\title{
Galerkin analysis of kinematic dynamos in the von Kármán geometry
}

\author{
L. Marié \\ Service de Physique de l'Etat Condensé, DSM/DRECAM/SPEC, CNRS/SPM URA 2464, CEA/Saclay, \\ 91191 Gif-sur-Yvette Cedex, France and Laboratoire de Physique des Océans, \\ Université de Bretagne Occidentale, BP 809, 29285 Brest, France \\ C. Normand ${ }^{\text {a) }}$ \\ Service de Physique Théorique, CEA/DSM/SPhT, CNRS/SPM URA 2306, CEA/Saclay, \\ 91191 Gif-sur-Yvette Cedex, France \\ F. Daviaud \\ Service de Physique de l'Etat Condensé, DSM/DRECAM/SPEC, CNRS/SPM URA 2464, CEA/Saclay, \\ 91191 Gif-sur-Yvette Cedex, France
}

(Received 15 February 2005; accepted 23 November 2005; published online 20 January 2006)

\begin{abstract}
We investigate dynamo action by solving the kinematic dynamo problem for velocity fields of the von Kármán type between two coaxial counter-rotating propellers in a cylinder. A Galerkin method is implemented that takes advantage of the symmetries of the flow and their subsequent influence on the nature of the magnetic field at the dynamo threshold. Distinct modes of instability have been identified that differ by their spatial and temporal behaviors. Our calculations give the result that a stationary and antisymmetric mode prevails at the dynamo threshold. We then present a quantitative analysis of the results based on the parametric study of four interaction coefficients obtained by reduction of our initially large eigenvalue problem. We propose these coefficients to measure the relative importance of the different mechanisms at play in the von Kármán kinematic dynamo.
\end{abstract}

(C) 2006 American Institute of Physics. [DOI: 10.1063/1.2158267]

\section{INTRODUCTION}

Fluid dynamos are a subject mainly referenced in the astrophysical or geophysical context, where the origin of magnetic fields in stellar or planetary objects such as the Sun or the Earth stands as a long-debated issue. Larmor ${ }^{1}$ was the first to suggest that a dynamo mechanism in the Sun could rely on internal fluid motion. Because these geo- and astrophysical applications involve spherical shells, a large number of theoretical or numerical approaches have focused on the spherical configuration. In the past decade, the achievement of dynamo experiments on a laboratory scale has raised a renewed interest for other geometries. The Riga experiment ${ }^{3}$ is based on the Ponomarenko model ${ }^{2}$ in which a conducting fluid flows with a helical motion inside a cylinder of infinite height and is at rest outside. The Karlsruhe experiment ${ }^{4}$ is based on the Roberts model ${ }^{5}$ in which the flow is organized in a spatially periodic array of helical motions. Besides these two experiments that have already given rise to dynamo action, there exist several attempts that have made the choice of a less constrained geometry. ${ }^{6-8}$ Here we are primarily concerned with the "Von Kármán Sodium" experiment" ${ }^{7,8}$ in which the fluid is confined in a cylindrical vessel of aspect ratio unity. The flow, generated by two counter-rotating propellers located at the ends of the cylinder, is of the von Kármán type. At the rotation rates used in the experiments the flow is highly turbulent, and its velocity fluctuations are of the same order of magnitude as the mean velocity. A few kinematic dynamo models have dealt with unsteady flows,

\footnotetext{
a) Author to whom correspondence should be addressed. Electronic mail: christiane.normand@cea.fr
}

like the modified ABC model of Galloway and Proctor, ${ }^{9}$ who considered chaotic flows, or the Ponomarenko dynamo revisited by Normand ${ }^{10}$ to take into account a time modulation of the helical flow. Nore et al. ${ }^{11}$ studied the dynamic dynamo problem in the Taylor-Green vortex. To begin with a simpler problem, a mean velocity field was extracted from experimental measurements and used as an input in the induction equation that was solved by numerical time integration. ${ }^{12,13}$ The present contribution intends to develop a complementary approach that can be viewed as the counterpart in cylindrical geometry of the work by Dudley and James ${ }^{14}$ for the spherical dynamo. A Galerkin analysis is performed to solve the induction equation when model flows with simple axial and radial dependencies are considered. The Galerkin method has significant advantages: it is faster than numerical integration, the symmetry properties of the flow can be used to simplify the problem, and the different modes of magnetic field are clearly identified. A similar analysis was performed recently by Livermore and Jackson, ${ }^{15}$ who considered the induction equation with spherical flows. Their results point out that energetic instability differs from eigenvalue or linear instability due to the non-self-adjointness of the induction equation.

A decisive advantage of the Galerkin approach in cases where it involves only a small number of trial functions is to allow for a parametric study of the matrix coefficients of the resulting eigenvalue problem. These coefficients can be assimilated to coupling coefficients related to the different physical phenomena contributing to the instability mechanism. When a large number of trial functions is required as in the present case, it is irrelevant to attribute a physical mean- 
ing to all the coefficients, and a reduction procedure must be undertaken as explained in Sec. VI to help understand the instability mechanisms at play in our dynamo problem.

The structure of the paper is as follows. Section II is devoted to the problem formulation. In Sec. III we emphasize the symmetry properties of the flow and show their incidence on the axial dependency of the magnetic field, which can be decomposed in two independent types of modes. In Sec. IV the Galerkin method is presented. The results are given in Sec. V and analyzed in Sec. VI through a method used to reduce large eigenvalue problems. Conclusions are drawn in Sec. VII.

\section{PROBLEM FORMULATION}

Kinematic dynamo theory investigates whether a given velocity field $\mathbf{V}$ is able to sustain dynamo action by solving the magnetic induction equation

$$
\frac{\partial \mathbf{B}}{\partial t}=\nabla \times(\mathbf{V} \times \mathbf{B})+\frac{1}{\mu \sigma} \Delta \mathbf{B},
$$

where $\mathbf{B}$ denotes the magnetic field with $\nabla \cdot \mathbf{B}=0, \mu$ and $\sigma$ are respectively the magnetic permeability and the electric conductivity of the considered medium, and $\Delta$ is the vector Laplacian operator. In our study, a flow of fluid of uniform conductivity $\sigma$ takes place in a cylinder of radius $R$ and height $h$, surrounded by an insulating medium that extends to infinity. The magnetic permeability is uniform over space. Choosing $R$ as the length scale, and introducing $L=h / 2 R$ as the aspect ratio of the cylinder, the variation ranges of the dimensionless cylindrical polar coordinates $(r, \varphi, z)$ are $(0$ $\leqslant r \leqslant 1,-\pi \leqslant \varphi \leqslant+\pi,-L \leqslant z \leqslant+L$ ). Introducing the magnetic Reynolds number $R_{m}=\mu \sigma \mathcal{U} R$, where $\mathcal{U}=|\mathbf{V}|_{\max }$ is the maximum value of the flow velocity, and choosing the magnetic field diffusion time $\mu \sigma R^{2}$ as the time scale, the induction equation is expressed in dimensionless form:

$$
\frac{\partial \mathbf{B}}{\partial t}=R_{m} \nabla \times(\mathbf{V} \times \mathbf{B})+\Delta \mathbf{B} .
$$

In this study, we have chosen to consider axisymmetric flows whose components $[u, v, w]$ could be expressed as products of independent radial and axial profiles:

$u(r, z)=\hat{u}(r) U(z), \quad v(r, z)=\hat{v}(r) V(z), \quad w(r, z)=\hat{w}(r) W(z)$.

The incompressibility condition $\nabla \cdot \mathbf{V}=0$ imposes that the radial and axial components of the velocity verify

$$
\frac{1}{r} \frac{\partial(r \hat{u})}{\partial r} U(z)+\hat{w} \frac{\partial W}{\partial z}=0 .
$$

Due to the flow axisymmetry, this equation does not involve the azimuthal component of the velocity $v$, which can thus be specified independently.

A second consequence of the flow axisymmetry is that the different azimuthal Fourier components of the magnetic field evolve independently. We can thus choose to consider only magnetic field modes of the form

$$
\mathbf{B}(\mathbf{r}, t)=a(t) \exp (\operatorname{im} \varphi) \mathbf{b}(r, z) .
$$

Modes with azimuthal wave number $m=0$ are always stable, as a consequence of Cowling's theorem. ${ }^{16}$ Moreover, since the properties of modes with $m<0$ can be deduced from those of modes with $m>0$ by complex conjugation, we have restricted our study to modes possessing azimuthal wave numbers $m \geqslant 1$.

Assuming for $\mathbf{V}$ and $\mathbf{B}$ the forms (3) and (5), respectively, and defining $b_{ \pm}=b_{r} \pm i b_{\varphi}$, we obtain the nondimensional induction equation as

$$
\begin{aligned}
\left(\mathcal{L}_{m \pm 1}-\sigma\right) b_{ \pm}= & R_{m}\left[\operatorname{iV}(z) S_{ \pm}\left(\hat{v}, b_{ \pm}\right)+U(z) C_{ \pm}\left(\hat{u}, b_{ \pm}\right)\right. \\
& \left.+\hat{w} W(z) \frac{\partial b_{ \pm}}{\partial z}-\left(\hat{u} \frac{d U}{d z} \pm i \hat{v} \frac{d V}{d z}\right) b_{z}\right] \\
\left(\mathcal{L}_{m}-\sigma\right) b_{z}= & R_{m}\left[i m \frac{\hat{v}}{r} V(z) b_{z}+\hat{u} U(z) \frac{\partial b_{z}}{\partial r}+\hat{w}\left(W(z) \frac{\partial b_{z}}{\partial z}\right.\right. \\
& \left.\left.-\frac{d W}{d z} b_{z}\right)-\frac{W(z)}{2} \frac{d \hat{w}}{d r}\left(b_{+}+b_{-}\right)\right]
\end{aligned}
$$

where $\mathcal{L}_{m}=\mathcal{D} * \mathcal{D}-m^{2} / r^{2}+\partial^{2} / \partial z^{2}$, with $\mathcal{D}=\partial / \partial r$ and $\mathcal{D}_{*}=\mathcal{D}$ $+1 / r$, and $\sigma=\sigma_{r}+i \sigma_{i}$ is the complex growth rate of the amplitude $a(t)=\exp (\sigma t)$. The following quantities have been introduced:

$$
\begin{aligned}
& S_{ \pm}\left(\hat{v}, b_{ \pm}\right)=m \frac{\hat{v}}{r} b_{ \pm} \pm \frac{1}{2}\left(\frac{\hat{v}}{r}-\frac{d \hat{v}}{d r}\right)\left(b_{+}+b_{-}\right), \\
& C_{ \pm}\left(\hat{u}, b_{ \pm}\right)=\left(\hat{u} \frac{\partial}{\partial r}-\frac{1}{2 r} \frac{\partial(r \hat{u})}{\partial r}\right) b_{ \pm}-\frac{r}{2} \frac{\partial}{\partial r}\left(\frac{\hat{u}}{r}\right) b_{\mp} .
\end{aligned}
$$

Our motivation for using $b_{ \pm}$instead of $b_{r, \varphi}$ in these equations is that doing so allows to decouple the components of the vector Laplacian, while requiring little extra work for the induction operator $\nabla \times(\mathbf{V} \times \mathbf{B})$.

In the insulating medium surrounding the flow, electric currents cannot flow, which implies that the magnetic field is irrotational. As it is also solenoidal, B satisfies the set of evolution equations in the insulating region: ${ }^{17}$

$$
\nabla \cdot \mathbf{B}=0, \quad \nabla \times \mathbf{B}=\mathbf{0} .
$$

In the case of spatially homogeneous magnetic permeability that we are considering, the magnetic field must also be continuous and finite over all space, and vanish for $r \rightarrow \infty$.

\section{INCIDENCE OF THE STRUCTURE OF THE FLOW ON THE MAGNETIC FIELD PROPERTIES}

\section{A. Axial structure of the velocity field}

We have shown in Fig. 1(a) a typical example of experimental velocity field measured with the help of the apparatus described in Refs. 12 and 18, using exactly counter-rotating turbines. We can see that both the poloidal and toroidal circulations are made of two counter-rotating cells. This flow is reminiscent of the $\left(s_{2}, t_{2}\right)$ flow considered by Dudley and James $^{14}$ in a spherical geometry. To capture in a simple way 

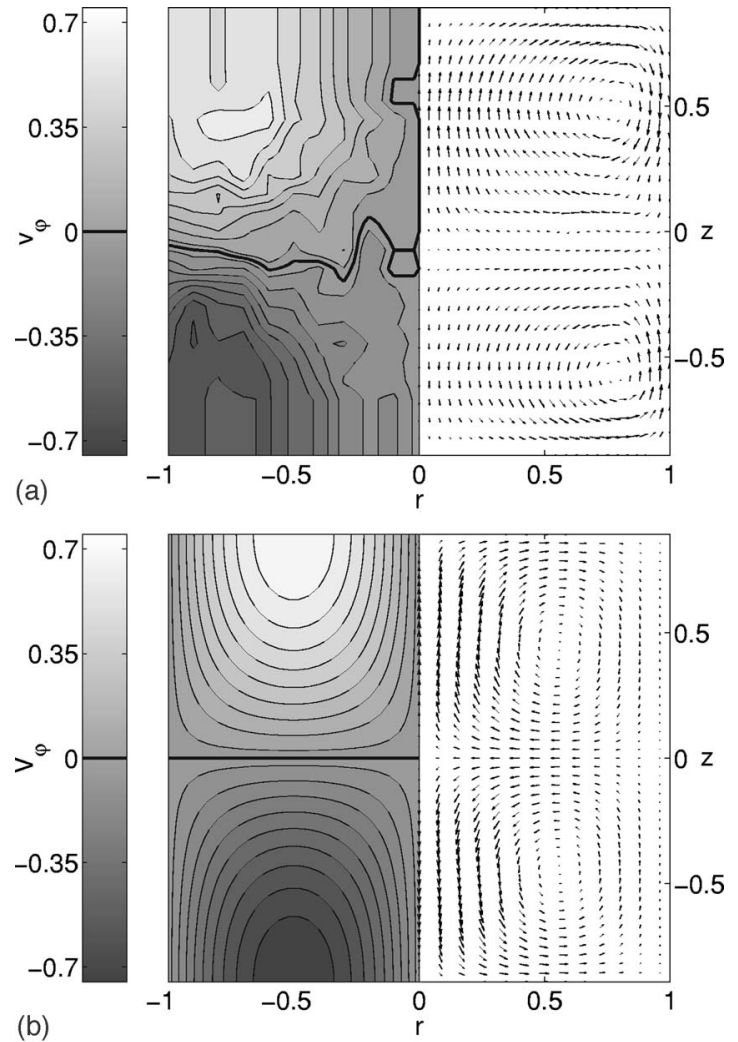

FIG. 1. (a) Typical example of experimental velocity field (see Ref. 12 for experimental details). (b) Typical example of velocity field used in this study [flow given by Eq. (56), namely]. In both cases, the toroidal (respectively, poloidal) component of the flow has been plotted on the left-hand side (respectively, right-hand side) of the graph, and the maximal magnitude of the velocity has been set to unity by proper rescaling. In both cases, the impeller located at the top (respectively, bottom) of the flow has a positive (respectively, negative) azimuthal velocity. Both rotate at the same frequency.

its main features, we have chosen to model the axial dependencies of the velocity components by the trigonometric expressions

$U(z)=\cos (\pi z / L), \quad V(z)=\sin (\pi z / 2 L), \quad W(z)=\sin (\pi z / L)$,

and varied the expressions used for the radial dependencies. Figure 1(b) represents as an example the velocity field obtained with the radial dependencies of Eqs. (56).

It was shown in Sec. II that performing the linear stability analysis of Eq. (2) involves solving Eqs. (10), which describe the evolution of the magnetic field in the insulating region. As in pseudospectral simulations, ${ }^{12}$ this particular stage of the study can be greatly simplified if one considers a "periodized" version of the problem, in which both the flow and the magnetic field are assumed periodic in the axial direction. Using as the unit cell the flow given by Eqs. (11) over the interval $z \in[-L ; L]$, though, introduces discontinuities in the azimuthal component of the flow, which are difficult to handle. To avoid this problem, we have thus chosen to use as a unit cell the whole range $z \in[-L ; 3 L]$, which is twice the original physical domain. Doing so yields velocity components that are continuous everywhere.

\section{B. Basic symmetry properties of the magnetic field eigenmodes}

It is well known that the symmetry properties of the flow strongly influence the structure of the magnetic field eigenmodes of Eqs. (2) and (10). For instance, the fact, noticed by Nore et al. in Ref. 19, that our model flow is left invariant by the rotation of an angle $\pi$ around any radius contained in the plane $z=0$ entails that its group of symmetry is the full group $O(2)$. According to Knobloch, ${ }^{20}$ this allows for the possibility that the bifurcation is steady. Flows not possessing this property, on the contrary, only have the smaller group of symmetry $S O(2)$, and bifurcate to nonaxisymmetric magnetic field states in a generically oscillatory way. (see Ref. 14 for examples of both cases).

Our modified problem, however, possesses more symmetry properties than the basic flow pattern defined over the interval $z \in[-L ; L]$. Namely, it is reflection symmetric across the plane $(z=L)$, and it is $4 L$ periodic in the axial direction. It is straightforward to check that, if the flow is invariant with respect to a symmetry operation $\mathcal{R}$, i.e., is such that $\mathcal{R}(\mathbf{V})$ $=\mathbf{V}$, then all the differential operators involved in Eqs. (2) and (10) commute with $\mathcal{R}$. It is then just as straightforward to check that, if $\mathcal{R}$ is such that $\mathcal{R} \circ \mathcal{R}=\mathbf{I d}$, all magnetic field eigenmodes of our problem can be sought as either symmetric [i.e., $\mathcal{R}(\mathbf{B})=\mathbf{B}$ ] or antisymmetric [i.e., $\mathcal{R}(\mathbf{B})=-\mathbf{B}$ ] with respect to $\mathcal{R}$. In our case, this implies that all the magnetic field eigenmodes can be obtained by searching independently for eigenmodes that are reflection symmetric or reflection antisymmetric across the plane $(z=L)$. The more complicated case of a magnetic field displaying a combination of the two behaviors does not need to be considered explicitly.

In order to make use of these properties, we shall in the following consider the Fourier decomposition of the magnetic field components, valid for a fixed value of the azimuthal wave number $m$ :

$$
\begin{aligned}
& b_{ \pm}(r, z)=\sum_{k=-\infty}^{\infty} A_{ \pm}^{k}(r) \exp (i k \pi z / 2 L), \\
& b_{z}(r, z)=\sum_{k=-\infty}^{\infty} A_{z}^{k}(r) \exp (i k \pi z / 2 L) .
\end{aligned}
$$

The requirement that $\mathbf{B}$ is symmetric with respect to $\mathbf{R}_{\mathbf{L}}$ can be expressed as

$$
b_{ \pm}(r, 2 L-z)=b_{ \pm}(r, z), \quad b_{z}(r, 2 L-z)=-b_{z}(r, z) .
$$

Replacing in these equations the components of $\mathbf{b}$ by their expressions (12), and collecting terms according to their wave numbers, one easily obtains that

$$
A_{ \pm}^{-k}(r)=(-1)^{k} A_{ \pm}^{k}(r), \quad A_{z}^{-k}(r)=(-1)^{k+1} A_{z}^{k}(r),
$$

and in particular that for $k=0$, the $z$ component vanishes:

$$
A_{z}^{0}(r)=0 .
$$

In a similar way, we can obtain the conditions satisfied by modes antisymmetric with respect to $\mathbf{R}_{\mathbf{L}}$ as 


$$
A_{ \pm}^{-k}(r)=(-1)^{k+1} A_{ \pm}^{k}(r), \quad A_{z}^{-k}(r)=(-1)^{k} A_{z}^{k}(r)
$$

and in this case for $k=0$ the \pm components vanish:

$$
A_{ \pm}^{0}(r)=0 .
$$

We shall make use of these relationships in Sec. IV E in order to obtain a system of equations involving positivewave-number Fourier components of the magnetic field alone.

\section{GALERKIN EXPANSION}

In the conducting medium, the axial dependencies of the velocity components couple the ordinary differential equations verified by the Fourier components of the magnetic field. Exact analytical resolution of such a system is currently beyond reach. We have thus carried out a Galerkin approximation of the amplitudes $A_{ \pm}^{k}(r), A_{z}^{k}(r)$ in order to obtain a more tractable algebraic system of equations. Following Backus, ${ }^{21}$ we have chosen to use as trial functions the solenoidal eigenmodes of the magnetic diffusion operator that satisfy insulating boundary conditions. In the following, we shall briefly recall the solution in the insulating medium and exhibit the trial and weighting functions we have used. We then orthogonalize the residual to the weighting functions to obtain the algebraic system of equations to be solved.

\section{A. Scalar products}

Given two vector functions of the $r$-variable $\mathbf{f}$ $=\left(f_{m+1}, f_{m-1}, f_{m}\right)$ and $\mathbf{g}=\left(g_{m+1}, g_{m-1}, g_{m}\right)$, we define their inner product by

$$
\langle\mathbf{f}, \mathbf{g}\rangle=\sum_{n=m-1}^{m+1} \int_{0}^{1} f_{n}^{*}(r) g_{n}(r) r d r
$$

where $f^{*}$ denotes the complex conjugate of $f$. Backus ${ }^{21}$ has shown that the diffusion operator is self-adjoint with respect to a similar inner product, in which the integral extends to infinity. As a consequence, this implies ${ }^{22}$ that its eigenmodes form a complete family, which spans the vector space of realizable magnetic fields. Our scalar product only involves the values of the functions for $r \leqslant 1$. This is convenient for computational purposes, but it turns out that the diffusion operator is not self-adjoint with respect to this inner product. Though the family of eigenmodes remains complete (i.e., a basis), it is no longer an orthogonal set, and we had to use a convenient set of weighting functions in the orthogonalization step of our Galerkin procedure.

\section{B. Magnetic field components in the insulating domain $r>1$}

Replacing in (10) the magnetic field components by their expressions (12), the radial dependencies of the $k \neq 0$ Fourier components of the magnetic field are readily obtained ${ }^{23}$ as

$$
A_{ \pm}^{k}(r)=i D_{k} \frac{k}{|k|} K_{m \pm 1}(|k| \pi r / 2 L),
$$

$$
A_{z}^{k}(r)=D_{k} K_{m}(|k| \pi r / 2 L),
$$

where $K_{n}(r)$ is the modified Bessel functions of order $n$. The amplitudes $D_{k}$ appearing in the above expressions are unknown coefficients to be determined later. For $k=0$, the only current-free solution is

$$
A_{+}^{0}=D_{0} r^{-(m+1)}, \quad A_{-}^{0}=A_{z}^{0}=0 .
$$

This Fourier component of the field is symmetric across the plane $(z=L)$. Antisymmetric eigenmodes are thus not allowed to possess a non-null, zero-wave-number magnetic field component outside the conducting domain.

\section{Trial and weighting functions for axial wave numbers $k \neq 0$}

Inside the cylinder, the following Galerkin approximation is used for the radial dependencies $A_{ \pm}^{k}(r)$ and $A_{z}^{k}(r)$ :

$$
A_{ \pm}^{k}(r)=i \frac{k}{|k|} \sum_{j=1}^{N} D_{j}^{k} \psi_{m \pm 1}^{k j}(r), \quad A_{z}^{k}(r)=\sum_{j=1}^{N} D_{j}^{k} \psi_{m}^{k j}(r) .
$$

The trial functions are defined as follows:

$$
\psi_{n}^{k j}=\frac{K_{n}(|k| \pi / 2 L)}{J_{n}\left(\mu_{k j}\right)} J_{n}\left(\mu_{k j} r\right), \quad n \in\{m \pm 1, m\},
$$

where $J_{n}(r)$ is the Bessel function of order $n \in\{m \pm 1, m\}$. These trial functions are eigenmodes of the magnetic diffusion operator for eigenvalues $-\left[\mu_{k j}^{2}+(k \pi / 2 L)^{2}\right]$ and satisfy the boundary conditions at $r=1$, i.e., they match continuously the exterior domain solutions (18) and (19). The coefficients $\mu_{k j}$ are determined by the condition that $\partial b_{r} / \partial r$ is also continuous at $r=1$, which stems from the $\nabla \cdot \mathbf{B}=0$ condition. When $k \neq 0$, the matching condition defining $\mu_{k j}$ is

$$
\begin{aligned}
\mu_{k j}( & \left.\frac{K_{m+1}(|k| \pi / 2 L)}{J_{m+1}\left(\mu_{k j}\right)}-\frac{K_{m-1}(|k| \pi / 2 L)}{J_{m-1}\left(\mu_{k j}\right)}\right) \\
& +\frac{|k| \pi}{L} \frac{K_{m}(|k| \pi / 2 L)}{J_{m}\left(\mu_{k j}\right)}=0 .
\end{aligned}
$$

For each $k$, there is an infinite set of real positive values $\mu_{k j}$, which we truncate to $N$, the number of radial modes in the Galerkin expansions (20). For a given value of $k$, the successive roots of (22) take increasingly large values as $j$ increases $\left(\mu_{k(j-1)}<\mu_{k j}\right)$. As an example, for $m=1$ and $k=1$, their values range between $\mu_{11}=2.217$ and $\mu_{1 j}=32.22$ for $j=20$.

In the standard Galerkin method, ${ }^{24}$ the weighting functions are chosen equal to the trial functions. In our case, where the trial functions happen not to form an orthogonal family, we have found it more convenient to use as weighting functions the eigenmodes of the adjoint magnetic field diffusion operator. It is a classical result ${ }^{22}$ that the eigenmodes of the adjoint problem (our weighting functions) form a family that is orthogonal to that of the eigenmodes of the direct problem (our trial functions). The weighting functions $\phi_{n}^{k j}$ are given by 


$$
\phi_{m \pm 1}^{k j}=\frac{1}{J_{m \pm 1}\left(\mu_{k j}\right)} J_{m \pm 1}\left(\mu_{k j} r\right), \quad \phi_{m}^{k j}=0 .
$$

The fact that their functional form is identical to that of the trial functions stems from the formal self-adjointness of the diffusion operator. The rather unconventional form of the boundary conditions of the direct problem, however, entails that the adjoint boundary conditions are very different. Use of these conditions yields the various amplitude coefficients and the equation defining the radial dependencies $\mu_{k j}$. This equation turns out to be identical to that obtained for the direct diffusion operator, as expected.

Using vector notations we define, respectively, $\boldsymbol{\Psi}_{j}^{k}$ $=\left(\psi_{m+1}^{k j}, \psi_{m-1}^{k j}, \psi_{m}^{k j}\right)$ and $\boldsymbol{\Phi}_{j}^{k}=\left(\phi_{m+1}^{k j}, \phi_{m-1}^{k j}, 0\right)$. It is worth noticing that the definition (20) of these functions implies that they are independent of the sign of $k$.

\section{The trial functions for the axial wave number $k=0$}

When $k=0$, the trial functions for $b_{ \pm}$and $b_{z}$ separate into two distinct sets:

$$
\begin{aligned}
& \psi_{m \pm 1}^{0 j}(r)=\frac{J_{m \pm 1}\left(\mu_{0 j} r\right)}{J_{m}\left(\mu_{0 j}\right)}, \quad \psi_{m}^{0 j}(r)=0, \\
& \psi_{m \pm 1}^{0 j}(r)=0, \quad \psi_{m}^{0 j}(r)=\frac{J_{m}\left(\bar{\mu}_{0 j} r\right)}{J_{m-1}\left(\bar{\mu}_{0 j}\right)},
\end{aligned}
$$

where the values of $\mu_{0 j}$ and $\bar{\mu}_{0 j}$ are solutions of $J_{m-1}\left(\mu_{0 j}\right)$ $=0$ and $J_{m}\left(\bar{\mu}_{0 j}\right)=0$, respectively. Thus two independent sets of coefficients $Z_{j}^{0}$ and $Y_{j}^{0}$ are required in the Galerkin expansions of $A_{ \pm}^{0}(r)$ and $A_{z}^{0}(r)$ :

$$
A_{ \pm}^{0}(r)=i \sum_{j=1}^{N} Z_{j}^{0} \psi_{m \pm 1}^{0 j}(r), \quad A_{z}^{0}(r)=\sum_{j=1}^{N} Y_{j}^{0} \psi_{m}^{0 j}(r) .
$$

As shown in Sec. III B, the first (respectively, second) expansion corresponds to symmetric (respectively, antisymmetric) modes. In the vector formulation, using the same notation for the two independent families of functions introduces no ambiguity. When symmetric modes are involved, the following definitions hold:

$$
\begin{aligned}
\boldsymbol{\Psi}_{j}^{0} & =\left(\psi_{m+1}^{0 j}, \psi_{m-1}^{0 j}, 0\right), \\
\boldsymbol{\Phi}_{j}^{0} & =\left(0, \psi_{m-1}^{0 j}, 0\right) .
\end{aligned}
$$

When antisymmetric modes are involved, the trial and weighting functions are

$$
\boldsymbol{\Psi}_{j}^{0}=\boldsymbol{\Phi}_{j}^{0}=\left(0,0, \psi_{m}^{0 j}\right) .
$$

In both cases, the weighting functions have been chosen equal to the eigenmodes of the adjoint diffusion operator, and their family is biorthogonal to that of the trial functions.

\section{E. Formation of the residuals}

Upon substituting the velocity profiles (11) and the Fourier representation of the magnetic field (12) into the governing equations (6) and (7) and collecting the terms that behave like $\exp (i k \pi z / 2 L)$, one gets, for each positive or negative value of $k$, the residuals

$$
\frac{R_{m}}{2}\left[T_{m \pm 1}^{k-1}+\bar{T}_{m \pm 1}^{k+1}+P_{m \pm 1}^{k-2}-\bar{P}_{m \pm 1}^{k+2}\right]-i\left(\sigma-L_{m \pm 1}^{k}\right) A_{ \pm}^{k}=\mathcal{R}_{ \pm}^{k},
$$

$$
\frac{R_{m}}{2}\left[T_{m}^{k-1}-T_{m}^{k+1}+P_{m}^{k-2}+\bar{P}_{m}^{k+2}\right]+\left(\sigma-L_{m}^{k}\right) A_{z}^{k}=\mathcal{R}_{z}^{k},
$$

where $L_{m}^{k}=\mathcal{D} * \mathcal{D}-m^{2} / r^{2}-(k \pi / 2 L)^{2}$. When $k \neq 0$, the quantities $T_{n}^{k}, \bar{T}_{n}^{k}$ and $P_{n}^{k}, \bar{P}_{n}^{k}$ are written as

$$
\begin{array}{ll}
T_{n}^{k}=\sum_{j} D_{j}^{k} \hat{T}_{n}^{k j}, & \bar{T}_{n}^{k}=\sum_{j} D_{j}^{k} \hat{T}_{n}^{-k j}, \\
P_{n}^{k}=\sum_{j} D_{j}^{k} \hat{P}_{n}^{k j}, & \bar{P}_{n}^{k}=\sum_{j} D_{j}^{k} \hat{P}_{n}^{-k j} .
\end{array}
$$

When $k=0$, they are replaced by the specific definitions

$$
\begin{aligned}
& T_{n}^{0}=\sum_{j} Y_{j}^{0} \hat{T}_{n}^{0 j}+\sum_{j} Z_{j}^{0} \mathcal{T}_{n}^{0 j}, \quad \bar{T}_{n}^{0}=\sum_{j} Y_{j}^{0} \hat{T}_{n}^{0 j}-\sum_{j} Z_{j}^{0} \mathcal{T}_{n}^{0 j}, \\
& P_{n}^{0}=\sum_{j} Y_{j}^{0} \hat{P}_{n}^{0 j}+\sum_{j} Z_{j}^{0} \mathcal{P}_{n}^{0 j}, \quad \bar{P}_{n}^{0}=\sum_{j} Y_{j}^{0} \hat{P}_{n}^{0 j}-\sum_{j} Z_{j}^{0} \mathcal{P}_{n}^{0 j} .
\end{aligned}
$$

Explicit expressions of the quantities $\hat{T}_{n}^{k j}, \hat{P}_{n}^{k j}$ and $\mathcal{T}_{n}^{k j}, \mathcal{P}_{n}^{k j}$ in terms of the trial functions and the radial profiles of the velocity components have been reported in the Appendix.

The aim of the Galerkin procedure ${ }^{24}$ is to reduce the system of equations (28) and (29) to an algebraic system for the unknown coefficients $Y_{j}^{0}, Z_{j}^{0}$, and $D_{j}^{k}$ with $k \neq 0$. Indeed, it will appear in the next section that one can restrict attention to the coefficients $D_{j}^{k}$ with strictly positive values of $k$ and then identify the unspecified coefficient $D_{j}^{0}$ to either $Y_{j}^{0}$ or $Z_{j}^{0}$ according to the symmetry of the modes under consideration. This restriction to a smaller system is made possible thanks to the relationships (14) and (16) that are representative of modes symmetric or antisymmetric with respect to the symmetry $\mathbf{R}_{\mathbf{L}}$. In the following it will be pertinent to consider separately the general case corresponding to $k>2$ and the special cases corresponding to the three values $k=0,1,2$.

\section{The general case: $k>2$}

In the case of symmetric modes having Fourier amplitudes satisfying (14), one deduces the following relationship for the coefficients $D_{j}^{k}$ of the Galerkin expansion:

$$
D_{j}^{-k}=(-1)^{k+1} D_{j}^{k} .
$$

It follows that the residuals for negative and positive values of $k$ are related through

$$
\mathcal{R}_{ \pm}^{-k}=(-1)^{k} \mathcal{R}_{ \pm}^{k}, \quad \mathcal{R}_{z}^{-k}=(-1)^{k+1} \mathcal{R}_{z}^{k},
$$

while in the case of antisymmetric modes, having Fourier coefficients satisfying (16), one deduces

$$
D_{j}^{-k}=(-1)^{k} D_{j}^{k}
$$

and consequently 


$$
\mathcal{R}_{ \pm}^{-k}=(-1)^{k+1} \mathcal{R}_{ \pm}^{k}, \quad \mathcal{R}_{z}^{-k}=(-1)^{k} \mathcal{R}_{z}^{k} .
$$

Hence, if after orthogonalization with the weighting functions the residuals $\mathcal{R}_{ \pm}^{k}$ and $\mathcal{R}_{z}^{k}$ are set equal to zero as required in the Galerkin procedure, it will also be true for $R_{ \pm}^{-k}$ and $R_{z}^{-k}$. Thus from now on we shall only consider positive values of $k$.

In vector formulation the residual $\mathbf{R}^{k}=\left(\mathcal{R}_{+}^{k}, \mathcal{R}_{-}^{k}, \mathcal{R}_{z}^{k}\right)$ is given by

$$
\begin{gathered}
\frac{R_{m}}{2} \sum_{j}\left(D_{j}^{(k-1)} \mathbf{T}_{j}^{(k-1)}+D_{j}^{(k+1)} \widetilde{\mathbf{T}}_{j}^{(k+1)}+D_{j}^{(k-2)} \mathbf{P}_{j}^{(k-2)}\right. \\
\left.+D_{j}^{(k+2)} \widetilde{\mathbf{P}}_{j}^{(k+2)}\right)+\sum_{j} D_{j}^{k}\left(\lambda_{k j}+\sigma\right) \mathbf{\Psi}_{j}^{k}=\mathbf{R}^{k}
\end{gathered}
$$

where $\lambda_{k j}=\mu_{k j}^{2}+(k \pi / 2 L)^{2}$. We have introduced the following quantities:

$$
\begin{aligned}
& \mathbf{T}_{j}^{k}=\left(\hat{T}_{m+1}^{k j}, \hat{T}_{m-1}^{k j}, \hat{T}_{m}^{k j}\right), \quad \widetilde{\mathbf{T}}_{j}^{k}=\left(\hat{T}_{m+1}^{-k j}, \hat{T}_{m-1}^{-k j},-\hat{T}_{m}^{k j}\right), \\
& \mathbf{P}_{j}^{k}=\left(\hat{P}_{m+1}^{k j}, \hat{P}_{m-1}^{k j}, \hat{P}_{m}^{k j}\right), \quad \widetilde{\mathbf{P}}_{j}^{k}=\left(-\hat{P}_{m+1}^{-k j},-\hat{P}_{m-1}^{-k j}, \hat{P}_{m}^{-k j}\right) .
\end{aligned}
$$

It is worth noticing that for $k>2$ the expression (38) for the residual is the same whether we are considering symmetric or antisymmetric modes. The differences arise when considering the cases $k=0,1,2$ on which emphasis is put in the next sections.

\section{The special case: $k=0$}

Letting $k=0$ in the general expressions (28) and (29), and taking into account the Galerkin expansions (24), one gets the residuals $\mathcal{R}_{ \pm}^{0}$ and $\mathcal{R}_{z}^{0}$ :

$$
\begin{aligned}
& \frac{R_{m}}{2} \sum_{j}\left[\left(D_{j}^{1}+D_{j}^{-1}\right) \hat{T}_{m \pm 1}^{-1 j}-\left(D_{j}^{2}-D_{j}^{-2}\right) \hat{P}_{m \pm 1}^{-2 j}\right] \\
& \quad+\sum_{j} Z_{j}^{0}\left(\mu_{0 j}^{2}+\sigma\right) \psi_{m \pm 1}^{0 j}(r)=\mathcal{R}_{ \pm}^{0}, \\
& \frac{R_{m}}{2} \sum_{j}\left[-\left(D_{j}^{1}-D_{j}^{-1}\right) \hat{T}_{m}^{1 j}+\left(D_{j}^{2}+D_{j}^{-2}\right) \hat{P}_{m}^{-2 j}\right] \\
& \quad+\sum_{j} Y_{j}^{0}\left(\bar{\mu}_{0 j}^{2}+\sigma\right) \psi_{m}^{0 j}(r)=\mathcal{R}_{z}^{0} .
\end{aligned}
$$

One can see that $Z_{j}^{0}$ will be coupled to $D_{j}^{1}$ and $D_{j}^{2}$ only if $\left(D_{j}^{1}+D_{j}^{-1}\right) \neq 0$ and $\left(D_{j}^{2}-D_{j}^{-2}\right) \neq 0$ that occurs only when considering the symmetric modes for which $D_{j}^{-k}=(-1)^{k+1} D_{j}^{-k}$. Conversely, $Y_{j}^{0}$ will be coupled to $D_{j}^{1}$ and $D_{j}^{2}$ only if $\left(D_{j}^{1}\right.$ $\left.-D_{j}^{-1}\right) \neq 0$ and $\left(D_{j}^{2}+D_{j}^{-2}\right) \neq 0$ that occurs only when considering the antisymmetric modes for which $D_{j}^{-k}=(-1)^{k} D_{j}^{-k}$. Hence the coefficients $Y_{j}^{0}$ and $Z_{j}^{0}$ cannot be present simultaneously in the algebraic system for the $D_{j}^{k}$ that implies that the original system breaks in two subsystems corresponding respectively to the symmetric or the antisymmetric modes, as expected on the basis of the symmetry arguments developed in Sec. III B.

The general expression (38) for the residual can still be used in the special case $k=0$, provided the general expressions (39) and (40) are slightly modified. First, it must be understood that $\mathbf{T}_{j}^{-1}=\mathbf{P}_{j}^{-2}=0$. The other changes, which de-
TABLE I. List of the quantities that have a different meaning according to the type of modes (symmetric or antisymmetric) considered.

\begin{tabular}{ccc}
\hline \hline$D_{j}^{0}$ & $Z_{j}^{0}$ (symmetric) & $Y_{j}^{0}$ (antisymmetric) \\
\hline $\boldsymbol{\Psi}_{j}^{0}, \boldsymbol{\Phi}_{j}^{0}$ & $\left(\psi_{m+1}^{0 j}, \psi_{m-1}^{0 j}, 0\right)$ & $\left(0,0, \psi_{m}^{0 j}\right)$ \\
$\mathbf{T}_{j}^{0}$ & $\left(\mathcal{T}_{m+1}^{0+1}, \mathcal{T}_{m-1}^{0 j}, 0\right)$ & $\left(\hat{T}_{m+1}^{0,}, \hat{T}_{m-1}^{0 j}, \hat{T}_{m}^{0 j}\right)$ \\
$\mathbf{P}_{j}^{0}$ & $\left(\mathcal{P}_{m+1}^{0 j}, \mathcal{P}_{m-1}^{0 j}, \mathcal{P}_{m}^{0 j}\right)$ & $\left(\hat{P}_{m+1}^{0 j}, \hat{P}_{m-1}^{0 j}, \hat{P}_{m}^{0 j}\right)$ \\
$\widetilde{\mathbf{T}}_{j}^{1}$ & $2\left(\hat{T}_{m+1}^{-1 j}, \hat{T}_{m-1}^{-1 j}, 0\right)$ & $2\left(0,0,-\hat{T}_{m}^{-1 j}\right)$ \\
$\tilde{\mathbf{P}}_{j}^{2}$ & $2\left(-\hat{P}_{m+1}^{-2 j},-\hat{P}_{m-1}^{-2 j}, 0\right)$ & $2\left(0,0, \hat{P}_{m}^{-2 j}\right)$ \\
\hline \hline
\end{tabular}

pend on the symmetry of the mode considered, can be stated as follows: for symmetric modes, we shall identify $D_{j}^{0}$ to $Z_{j}^{0}$, and define

$$
\widetilde{\mathbf{T}}_{j}^{1}=2\left(\hat{T}_{m+1}^{-1 j}, \hat{T}_{m-1}^{-1 j}, 0\right), \quad \widetilde{\mathbf{P}}_{j}^{2}=2\left(-\hat{P}_{m+1}^{-2 j},-\hat{P}_{m-1}^{-2 j}, 0\right) .
$$

For antisymmetric modes, we shall identify $D_{j}^{0}$ to $Y_{j}^{0}$, and define

$$
\widetilde{\mathbf{T}}_{j}^{1}=2\left(0,0,-\hat{T}_{m}^{-1 j}\right), \quad \widetilde{\mathbf{P}}_{j}^{2}=2\left(0,0, \hat{P}_{m}^{-2 j}\right) .
$$

\section{The special cases: $k=1,2$}

When $k=1$, in Eq. (38), the term $D_{j}^{0} \mathbf{T}_{j}^{0}$ requires special attention since $D_{j}^{0}$ can be assimilated to either $Y_{j}^{0}$ or $Z_{j}^{0}$ according to the type of modes (symmetric or antisymmetric) considered and the definition of $\mathbf{T}_{j}^{0}$ differs in the two cases. For $k=2$ the same problem arises with the term $D_{j}^{0} \mathbf{P}_{j}^{0}$. The appropriate expressions of $\mathbf{T}_{j}^{0}, \mathbf{P}_{j}^{0}$ can be found in Table I for each type of modes.

\section{F. Orthogonalization with the weighting functions}

The Galerkin method is implemented by constructing for each value of $k(0 \leqslant k \leqslant M)$ the inner product of the residual with the weighting functions $\boldsymbol{\Phi}_{i}^{k}(1 \leqslant i \leqslant N)$ and requiring $\left\langle\boldsymbol{\Phi}_{i}^{k}, \mathbf{R}^{k}\right\rangle=0$. One obtains an algebraic system for each set of coefficients $\left\{D_{j}^{k}\right\}$ corresponding to either the symmetric modes or the antisymmetric modes

$$
\begin{aligned}
\frac{R_{m}}{2} \sum_{j=1}^{N} & {\left[D_{j}^{(k-1)}\left\langle\boldsymbol{\Phi}_{i}^{k}, \mathbf{T}_{j}^{(k-1)}\right\rangle+D_{j}^{(k+1)}\left\langle\boldsymbol{\Phi}_{i}^{k}, \widetilde{\mathbf{T}}_{j}^{(k+1)}\right\rangle\right.} \\
& \left.+D_{j}^{(k-2)}\left\langle\boldsymbol{\Phi}_{i}^{k}, \mathbf{P}_{j}^{(k-2)}\right\rangle+D_{j}^{(k+2)}\left\langle\boldsymbol{\Phi}_{i}^{k}, \widetilde{\mathbf{P}}_{j}^{(k+2)}\right\rangle\right] \\
& +\sum_{j=1}^{N} D_{j}^{k}\left(\lambda_{k j}+\sigma\right)\left\langle\boldsymbol{\Phi}_{i}^{k}, \boldsymbol{\Psi}_{j}^{k}\right\rangle=0 .
\end{aligned}
$$

Though Eq. (45) gives a unified description of the two subsystems they are not identical. Their differences already pointed out in Secs. IV E 2 and IV E 3 when examining the cases $k=0,1,2$ are recapitulated in Table I.

\section{G. Numerical implementation}

The algebraic system (45) is equivalent to a generalized eigenvalue problem for the growth rate $\sigma: \mathbf{M}_{A} \mathbf{X}=\sigma \mathbf{M}_{B} \mathbf{X}$, 
where $\mathbf{M}_{A}$ and $\mathbf{M}_{B}$ are large matrices of order $N(M+1)$ and $\mathbf{X}=\left(D_{1}^{0}, \ldots, D_{N}^{0}, \ldots, D_{1}^{M}, \ldots, D_{N}^{M}\right)$ is the vector of unknown coefficients $D_{i}^{k}$.

The nondiagonal elements of $\mathbf{M}_{A}$ are expressed as an integral over the $r$ variable multiplied by $R m$. These integrals that are related to the quantities surrounded with brackets in Eq. (45) have to be computed once and are then stored. The same type of calculation was done in Ref. 15 for spherical dynamos.

Due to the orthogonality properties mentioned in Secs. IV C and IV D, $\mathbf{M}_{B}$ is diagonal, and can be set equal to the identity through proper normalization of the trial functions so that one is led to a standard eigenvalue problem ( $\mathbf{M}$ $-\sigma \mathbf{I}) \mathbf{X}=0$, where $\mathbf{I}$ is the identity matrix of order $N(M+1)$.

We have implemented two different versions of the method, one in MATLAB and the other in FORTRAN. The MATLAB version was primarily used for visualization of the magnetic field modes and plotting, while the FORTRAN version, being both more accurate and more efficient, was used as a reference implementation, and in the search for the critical values of the magnetic Reynolds number for the different flows (see the following section). We have carefully checked that the results obtained with both codes are identical, and that they compare favorably with those obtained using a time-stepping pseudospectral code. ${ }^{12}$

In both codes, most of the integrals involved in the construction of the matrices $\mathbf{M}_{A}$ and $\mathbf{M}_{B}$ were evaluated numerically through the use of Gaussian quadrature formulas, but analytical expressions were used in the FORTRAN code when available.

NAG library subroutines were used by the FORTRAN code in the solution of the algebraic eigensystem, while the MATLAB code relied on MATLAB's implementation of Arnoldi's method.

It is worth noticing here that the symmetries of the flow under consideration bear consequences on the properties of the matrices $\mathbf{M}_{A}$ and $\mathbf{M}_{B}$. In particular, the $O(2)$ symmetry of the flow implies that both matrices are real valued, although they describe the evolution of complex components of the magnetic field. Hence, the eigenvalues $\sigma$ are either real or they appear in complex conjugate pairs for each azimuthal Fourier component of the magnetic field. ${ }^{20}$

We focus on the eigenvalue whose real part, $\sigma_{r}$, has the lowest absolute value since we are interested in the critical value $R_{m c}$ for which $\sigma_{r}$ goes through zero. As the value of $R_{m}$ increases, it becomes more and more difficult to insure convergence of the results. Consequently, the number of axial modes $M$ and the number of radial modes $N$ need to be increased up to $M=21$ and $N=30$ for the largest values of $R_{m}$ we have considered. In these limiting cases the size of the matrices $\mathbf{M}_{A}$ and $\mathbf{M}_{B}$ exceeds $600 \times 600$.

\section{RESULTS}

To remain close to the experimental conditions we have chosen to consider a cylinder of aspect ratio unity $(L=1)$. Our results are presented for very simple flows with radial profiles of the velocity components given by polynomial expressions. These profiles always satisfy the impermeability
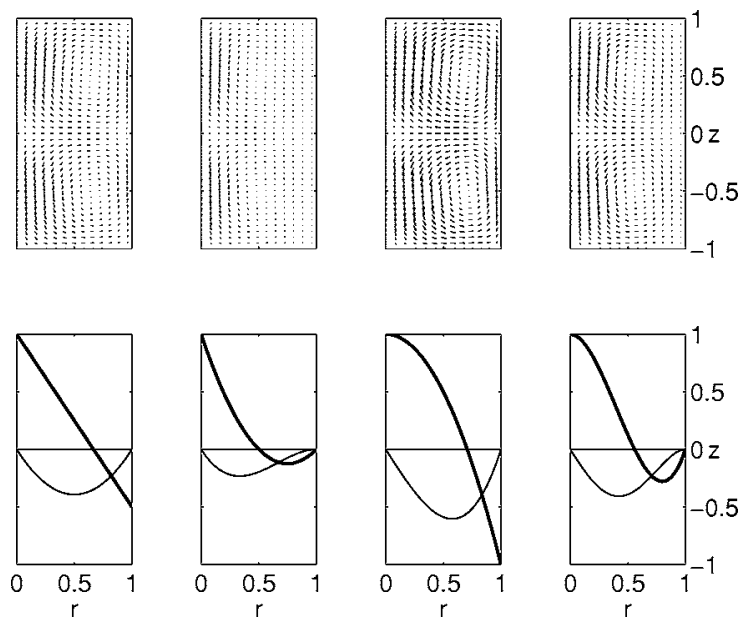

FIG. 2. Top: Poloidal velocity fields used in the study. From left to right: $\Psi=r(1-r), \Psi=r(1-r)^{2}, \Psi=r\left(1-r^{2}\right), \Psi=r(1-r)^{2}(1+2 r)$. Bottom: Corresponding radial profiles $\hat{u}(r)$ (thin black lines) and $\hat{w}(r)$ (thick black lines), normalized with $\hat{w}(0)=1$.

boundary condition $\hat{u}(1)=0$, but not necessarily the full noslip conditions $\hat{v}(1)=\hat{w}(1)=0$. The toroidal component $\hat{v}(r)$ is chosen independently of the poloidal components $\hat{u}(r)$, $\hat{w}(r)$ that, according to the continuity equation (4), can be expressed in terms of the streamfunction $\Psi(r)$ :

$$
\hat{u}(r)=-\frac{\pi}{L} \Psi, \quad \hat{w}(r)=\frac{1}{r} \frac{\partial}{\partial r}(r \Psi) .
$$

Introducing the mean values of the poloidal and toroidal velocities as follows:

$$
\left.\begin{array}{l}
\bar{U}_{\mathrm{pol}}=\int_{0}^{L} d z \int_{0}^{1}\left[u(r, z)^{2}+w(r, z)^{2}\right]^{1 / 2} r d r \\
\bar{U}_{\mathrm{tor}}=\int_{0}^{L} d z \int_{0}^{1}|v(r, z)| r d r
\end{array}\right\} \quad \text { and } \Gamma=\frac{\bar{U}_{\mathrm{pol}}}{\bar{U}_{\mathrm{tor}}}
$$

the flow can be specified by its poloidal to toroidal ratio $\Gamma$.

Four families of flows are considered. Each family is characterized by a fixed poloidal circulation that is assumed to satisfy some prescribed boundary conditions and is associated to different radial profiles of the toroidal velocity. The different poloidal circulations have been represented in Fig. 2. Starting in Sec. V B with the simplest case corresponding to a free-slip boundary at $r=1$, more constraints are progressively added to end up in Sec. VE with a velocity field satisfying the no-slip conditions on $r=1$ and having a smooth behavior on $r=0$.

As diffusion effects tend to strongly stabilize modes possessing large azimuthal wave numbers, and Cowling's theorem shows that axisymmetric eigenmodes cannot reach criticality, we have chosen to restrict our study to modes with $m=1$ azimuthal dependencies. A complementary study of modes possessing more complex angular structures is left for future work. 


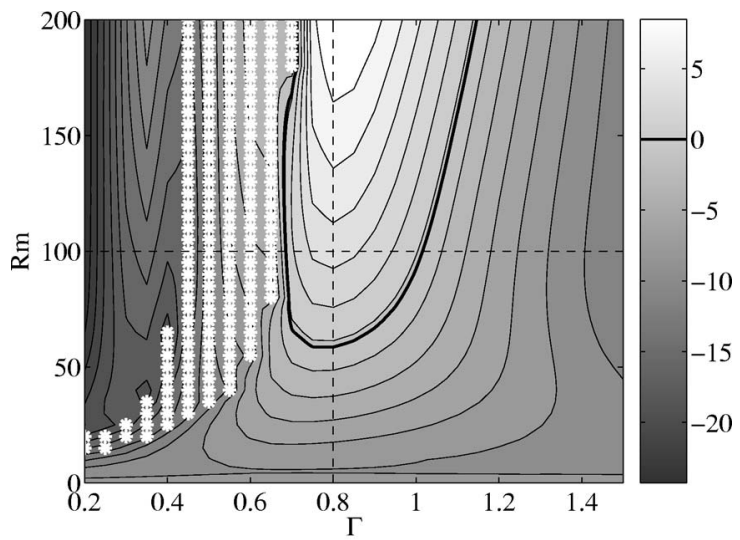

FIG. 3. Isocontour plot of the leading (i.e., least negative) magnetic field growth rate as a function of $R_{m}$ and $\Gamma$, obtained for the velocity field given by Eq. (56). The thick black line is the neutral stability curve. In the region marked with white stars, the leading eigenvalue is imaginary. Only eigenvalues corresponding to antisymmetric modes have been represented. The dashed lines represent the directions along which the data of Figs. 4(a) and 4(b) have been obtained.

\section{A. General results}

In this section we present some results of general applicability, using data obtained in the special case of the velocity field studied in Sec. V E. A first point worth noticing is that for all the profiles considered here it is always a stationary dynamo (with $\sigma_{i}=0$ ) that sets in at threshold as shown in Figs. 3, 4(a), and 4(b). However, below the dynamo threshold and for low values of the poloidal to toroidal ratio $\Gamma$, damped oscillatory modes can occur as shown on Fig. 4(b), where the real part $\sigma_{r}$ of the growth rate is drawn as a function of $\Gamma$. Solid lines correspond to stationary modes with real eigenvalues $\sigma=\sigma_{r}$ and dotted lines correspond to oscillatory modes with complex eigenvalues $\left(\sigma=\sigma_{r} \pm \sigma_{i}\right)$. For a fixed value of $\Gamma$, the eigenvalue spectrum is plotted in Fig. 4(a) as a function of the magnetic Reynolds number. Starting at $R_{m}=0$, all eigenvalues are purely real. They then evolve with increasing values of $R_{m}$, giving rise to complex eigenvalues by the collapse of pairs of real eigenvalues. This merging process never concerns the least negative eigenvalue that remains real for all values of $R_{m}$ and changes its sign when the threshold value $R_{m c}$ is reached. Another interesting point is that the first magnetic field eigenmode is always antisymmetric. We have gathered in Fig. 5 the eigenvalue spectra obtained separately for symmetric and antisymmetric eigenmodes. It can be seen clearly that the leading eigenvalues associated with both types of modes follow very similar paths, but that the leading antisymmetric mode is the first to achieve criticality. We have consistently observed this behavior for all the velocity profiles we have studied. Visual observation of the two critical modes shows that their structures are very similar over the interval $-L$ $\leqslant z \leqslant L$, though their symmetry properties differ with respect to $\mathbf{R}_{\mathbf{L}}$. In the following, only data corresponding to antisymmetric eigenmodes will be presented.

The spatial characteristics of the magnetic field above threshold are illustrated in Fig. 6, where the poloidal contri-
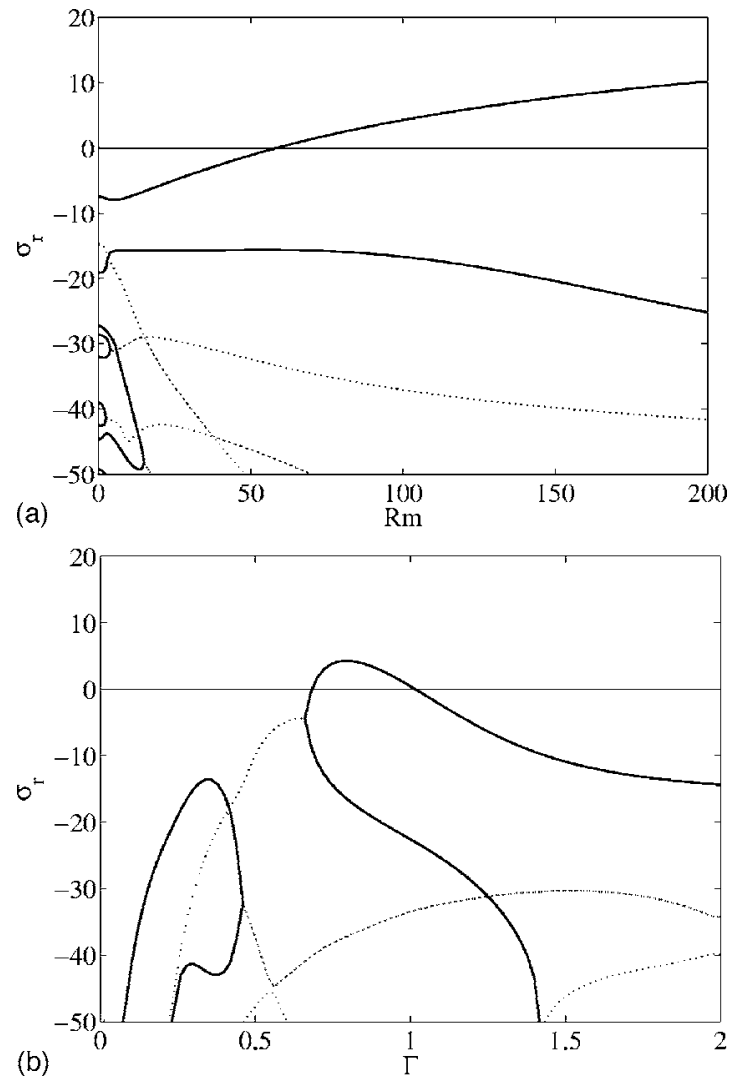

FIG. 4. Leading part of the magnetic field evolution operator spectrum, obtained for the velocity field given by Eq. (56). (a) The value of $\Gamma$ is kept constant and equal to $0.8, R_{m}$ varies. (b) The value of $R_{m}$ is kept constant and equal to $100, \Gamma$ varies. On both graphs, real eigenvalues are represented as continuous lines, and complex-conjugate eigenvalue pairs are represented as dashed lines. The complex-conjugate eigenvalues that seem to originate from $R_{m}=0, \sigma_{r} \simeq-15$ on graph (a) are distinct and real on the $R_{m}=0$ axis, and collapse very shortly afterwards. Only eigenvalues corresponding to antisymmetric modes have been represented.

bution $\mathbf{B}_{p}=\left(B_{r}, B_{z}\right)$ and the toroidal contribution $B_{\varphi}$ of a representative antisymmetric eigenmode have been plotted. They admit the following decomposition:

$$
\mathbf{B}_{p}=\sin \varphi \mathbf{B}_{p 1}(r, z)+\cos \varphi \mathbf{B}_{p 2}(r, z),
$$

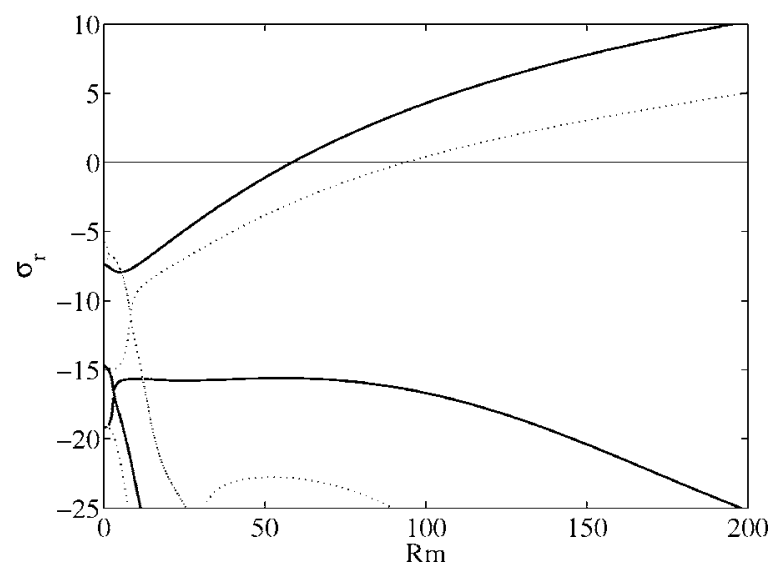

FIG. 5. Leading part of the magnetic field evolution operator spectrum, obtained for the velocity field given by Eq. (56), for a fixed value of $\Gamma$ equal to 0.8 . The continuous (respectively, dotted) lines denote eigenvalues associated to modes that are antisymmetric (respectively, symmetric) with respect to $\mathbf{R}_{\mathbf{L}}$. 


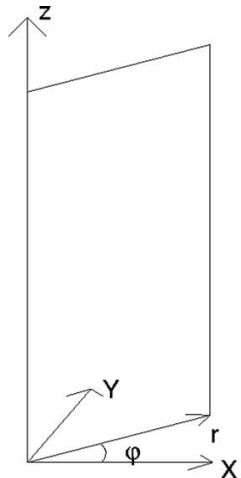

(a)
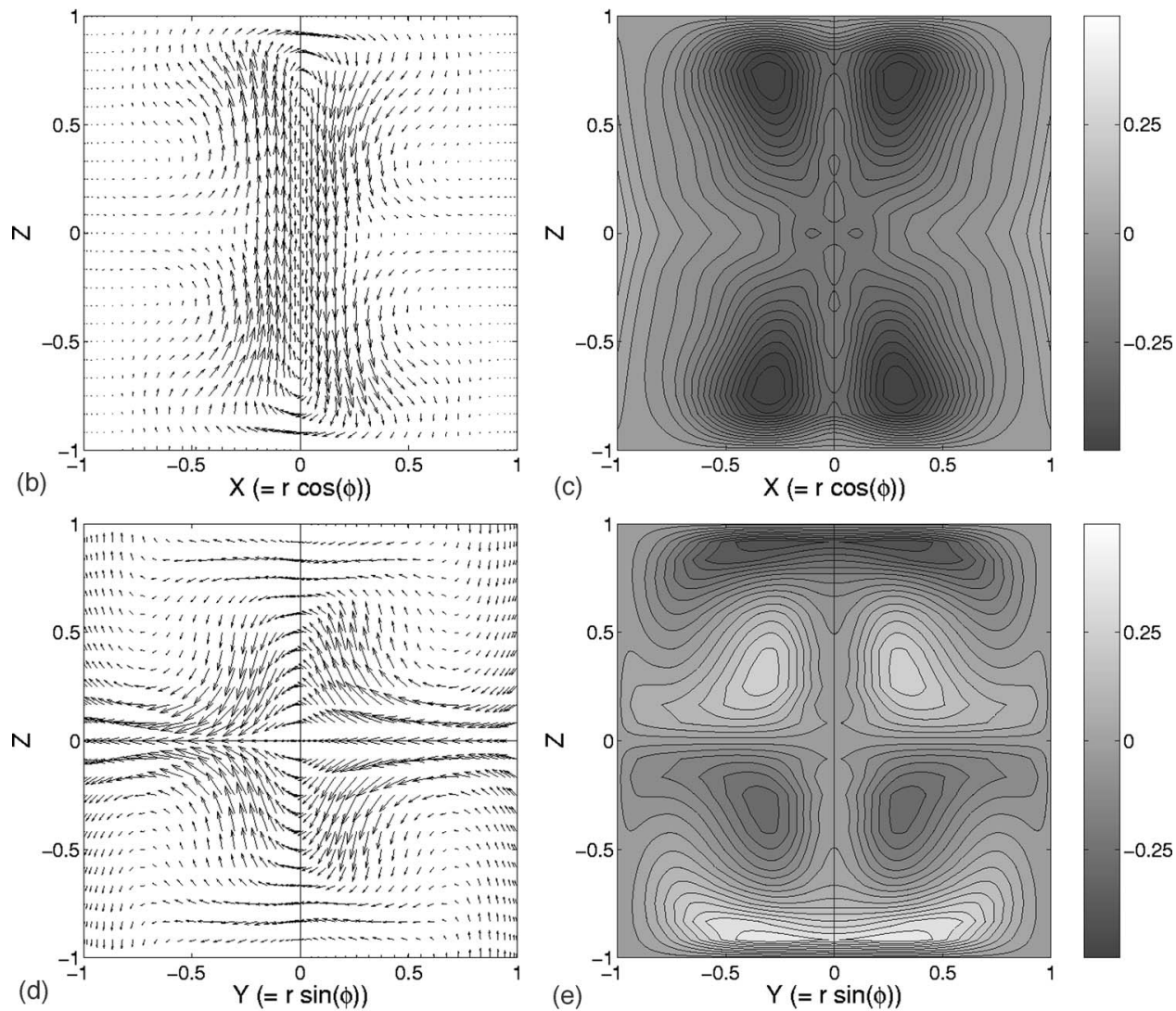

FIG. 6. Leading mode of magnetic field for the velocity field given by Eq. (56) for $R_{m}=100, \Gamma=0.8$. (a) Sketch of the Cartesian coordinates used in the graphs. (b) Poloidal component of the magnetic field in the plane $(\mathrm{Ox}, \mathrm{Oz})$ $(\sin \varphi=0)$. (c) Azimuthal component of the magnetic field in the plane $(\mathrm{Ox}$, Oz). (d) Poloidal component of the magnetic field in the plane $(\mathrm{Oy}, \mathrm{Oz})$ $(\cos \varphi=0)$. (e) Azimuthal component of the magnetic field in the plane (Oy, $\mathrm{Oz})$.

$$
B_{\varphi}=\cos \varphi B_{\varphi 1}(r, z)+\sin \varphi B_{\varphi 2}(r, z) .
$$

Cartesian coordinates are introduced in Fig. 6, where the plane $(O X, O Z)$ corresponds to $\sin \varphi=0$ and the plane $(O Y, O Z)$ to $\cos \varphi=0$. Figures 6(b) and 6(d) represent the poloidal part of the magnetic field drawn in the two orthogonal diametral planes, respectively, $(O X, O Z)$ and $(O Y, O Z)$. Figures $6(\mathrm{c})$ and $6(\mathrm{e})$ correspond to the toroidal part in the same diametral planes. The main features of these drawings compare remarkably well with the results of pseudospectral simulations obtained in the same conditions as was checked in Ref. 18. They also compare favorably with the results obtained for an experimental velocity profile and a slightly higher magnetic Reynolds number. ${ }^{12}$

We have represented in Fig. 7 the leading oscillating magnetic field mode obtained in the same setting, though for $R_{m}=100$ and $\Gamma=0.55$. We can see that its regions of high amplitude are shifted significantly towards the impeller located to the top. The complex character of its associated eigenvalue induces it to rotate as a whole around the cylinder axis, in the same direction as the impeller. The image of this mode by rotation $\mathbf{R}_{\pi}$, which has similar characteristics but lies close to the bottom impeller, is associated to the complex conjugate eigenvalue. These modes possess structures quite different from that of the critical mode obtained for higher values of $\Gamma$, and their dynamics probably involves different mechanisms. We have also consistently observed them to be significantly more stable. We thus do not expect them to be capable of self-exciting for achievable values of the magnetic Reynolds number (Ravelet et al. ${ }^{13}$ report a self-excitation 

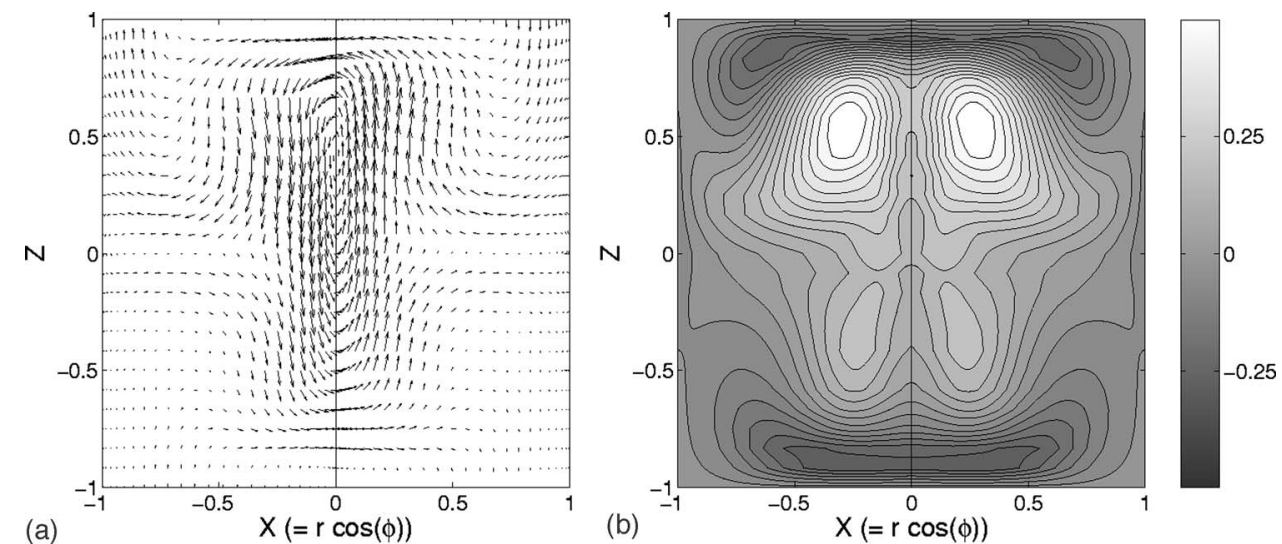

FIG. 7. Leading oscillating mode of magnetic field for the velocity field given by Eq. (56) for $R_{m}=100, \Gamma$ $=0.55$, represented in the same coordinates. (a) Poloidal component of the magnetic field in the plane $(\mathrm{Ox}, \mathrm{Oz})$ $(\sin \varphi=0)$. (b) Azimuthal component of the magnetic field in the plane (Ox, $\mathrm{Oz}$ ). (c) Poloidal component of the magnetic field in the plane $(\mathrm{Oy}, \mathrm{Oz})$ $(\cos \varphi=0)$. (d) Azimuthal component of the magnetic field in the plane (Oy, $\mathrm{Oz})$.

N

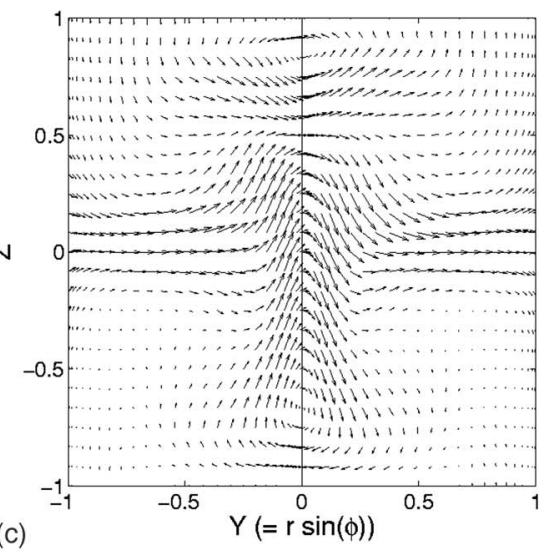

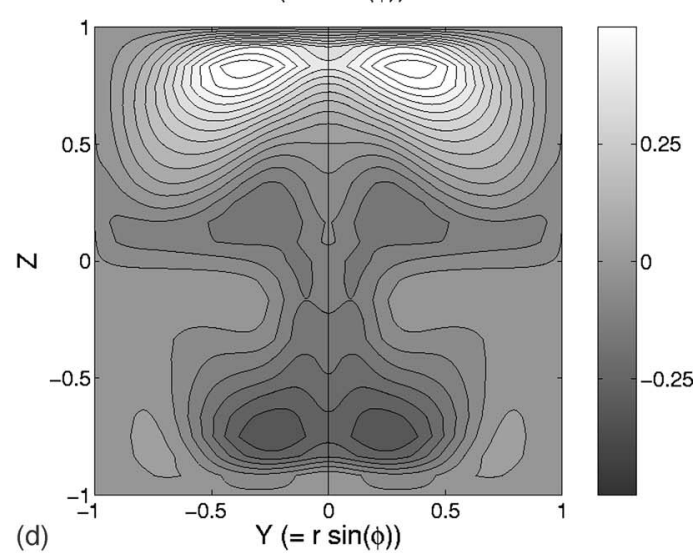

threshold of 215 for such modes in pseudospectral simulations using experimentally measured velocity fields).

\section{B. Poloidal circulation with $\Psi=r(1-r)$}

The simplest choice is to consider the radial profiles of the velocity components given by

$$
\begin{aligned}
& \hat{u}(r)=-\frac{\pi}{2 L} r(1-r), \\
& \hat{w}(r)=1-3 r / 2, \\
& \hat{v}(r)=\varepsilon r,
\end{aligned}
$$

with the impermeability condition $\hat{u}(1)=0$ as the only constraint. The corresponding poloidal circulation has been represented in Fig. 2(a). Here and in the following the poloidal velocity is chosen such that $\max \{\hat{u}(r), \hat{w}(r)\}$ is equal to unity. The parameter $\varepsilon=\max \{\hat{v}(r)\}$ allows to vary in a simple way the poloidal-to-toroidal ratio $\Gamma$. According to our definition of the magnetic Reynolds number the velocity must be normalized so that the maximum value of $\left[u(r, z)^{2}+v(r, z)^{2}\right.$ $\left.+w(r, z)^{2}\right]^{1 / 2}$ is equal to unity. The maximum over $z$ arises for either $z= \pm L$, where $U_{\max }=\max \left[\hat{u}^{2}(r)+\hat{v}^{2}(r)\right]^{1 / 2}$ or for $z$ $= \pm L / 2$, where $U_{\max }=\max \left[\hat{w}^{2}(r)+\hat{v}^{2}(r) / 2\right]^{1 / 2}$. For each value of $\varepsilon$, the normalization factor $U_{\max }$ is calculated and the computations are performed with the normalized radial velocity profiles $(\widetilde{u}, \widetilde{v}, \widetilde{w})=U_{\max }^{-1}(\hat{u}, \hat{v}, \hat{w})$. Once $\varepsilon$ is fixed, the spatial structure of the flow is frozen. Then, varying the magnetic Reynolds number allows to change the strength of the flow. Hence, the magnetic Reynolds number and the poloidal-to- toroidal ratio $\Gamma \sim 1 / \varepsilon$ can be varied independently. The magnetic Reynolds number $R_{m 0}$ corresponding to marginal stability $\left(\sigma_{r}=0\right)$ is obtained as a function of $\varepsilon$ or equivalently $\Gamma$. The neutral curve $R_{m 0}(\Gamma)$, a typical example of which is plotted in Fig. 3, exhibits a minimum for a particular value $\Gamma_{c}$. The critical magnetic Reynolds number is defined as $R_{m c}=R_{m 0}\left(\Gamma_{c}\right)$. With the radial profiles (50) and (51), the critical value $R_{m c}=149$ is reached for $\varepsilon_{c}=1.15$ or $\Gamma_{c}=0.602$. The maximum value of the velocity occurs at the edge of the top and bottom boundaries $\left(z_{\max }= \pm L, r_{\max }=1\right)$, with $U_{\max }=\varepsilon$.

To satisfy the additional constraint: $\hat{v}(1)=0$, the azimuthal velocity profile (51) is then replaced by

$$
\hat{v}=4 \varepsilon r(1-r) .
$$

With the poloidal components $\hat{u}(r), \hat{w}(r)$ still given by (50), this leads to $R_{m c}=62$ for $\varepsilon_{c}=0.92$ or $\Gamma_{c}=0.756$. In this case, the maximum value of the velocity occurs at midradius of the top and bottom boundary $\left(z_{\max }= \pm L, r_{\max }=0.5\right)$ while $U_{\max }=\left[\varepsilon^{2}+(\pi / 8)^{2}\right]^{1 / 2}$. Hence, changing the radial behavior of the toroidal velocity and in particular the location of its maximum value that is shifted from $r=1$ in (50) to $r=0.5$ in (52) has the consequence of lowering the critical magnetic Reynolds number by a factor of greater than 2 .

More generally, azimuthal velocity profiles of the type $\hat{v}(r)=\varepsilon v_{n}(r)$ with for $n \geqslant 2$,

$$
v_{n}(r)=A_{n} r\left(1-r^{(n-1)}\right) \quad \text { and } A_{n}=\frac{n^{n /(n-1)}}{(n-1)},
$$

have been considered in association with the poloidal circulation (50). The profiles $v_{n}(r)$ in (53) are normalized to unity. 
TABLE II. Values of $R_{m c}, \Gamma_{c}$ and $\varepsilon_{c}$ for different azimuthal velocity profiles $\hat{v}=\varepsilon_{c} v_{n}(r)$ and a poloidal streamfunction $\Psi=r(1-r)$.

\begin{tabular}{cccc}
\hline \hline$v_{n}(r)$ & $R_{m c}$ & $\Gamma_{c}$ & $\varepsilon_{c}$ \\
\hline$r$ & 149 & 0.602 & 1.15 \\
$r\left(1-r^{9}\right)$ & 68 & 0.672 & 0.96 \\
$r\left(1-r^{5}\right)$ & 62 & 0.688 & 0.94 \\
$r\left(1-r^{2}\right)$ & 60 & 0.731 & 0.91 \\
$r(1-r)$ & 62 & 0.756 & 0.92 \\
$r\left(1-r^{1 / 2}\right)$ & 66 & 0.781 & 0.92 \\
\hline \hline
\end{tabular}

For each profile, the critical values of the magnetic Reynolds number and the associated values of $\Gamma_{c}$ are listed in Table II. The values of $R_{m c}$ are plotted in Fig. 8 for the corresponding values of $\Gamma_{c}$. The lowest threshold for dynamo action is obtained when the toroidal circulation is represented by the profile $v_{3}$ and corresponds to $R_{m c}=60$ and $\Gamma_{c}=0.731$.

\section{Poloidal circulation with $\Psi=r(1-r)^{2}$}

The no-slip conditions $\hat{u}(1)=\hat{w}(1)=0$ are satisfied by the following poloidal circulation:

$$
\begin{aligned}
& \hat{u}(r)=-\frac{\pi}{2 L} r(1-r)^{2}, \\
& \hat{w}(r)=(1-r)(1-2 r)
\end{aligned}
$$

[see Fig. 2(b)]. When combined to the profile (52) for $\hat{v}(r)$, this leads to $R_{m c}=112$ for $\varepsilon_{c}=0.36$ or $\Gamma_{c}=0.791$. In this case, the maximum value of the velocity occurs on the cylinder axis $\left(z_{\max }= \pm L / 2, r_{\max }=0\right)$ and $U_{\max }=\hat{w}(0)=1$ is independent of $\varepsilon$. Changing the poloidal components of the flow from (50)-(54) has the consequence to increase the critical magnetic Reynolds number. This tendency is confirmed by the results shown in Table III, where the values of $R_{m c}$ and $\Gamma_{c}$ corresponding to a selection of azimuthal velocity profiles are reported. The threshold for dynamo action is minimum when $\hat{v}(r)=\varepsilon(4 / 27) r(1-r)^{2}$, with $R_{m c}=85$, a higher value

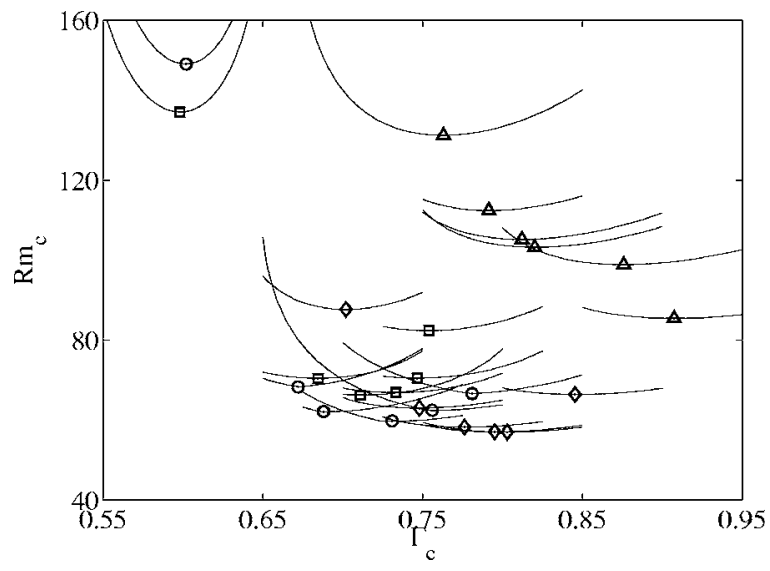

FIG. 8. Critical values of the magnetic Reynolds number and poloidal-totoroidal ratio for the different velocity fields used in the study. $(\bigcirc): \Psi$ $=r(1-r),(\triangle): \Psi=r(1-r)^{2},(\square): \Psi=r\left(1-r^{2}\right),(\diamond): \Psi=r(1-r)^{2}(1+2 r)$. The corresponding neutral stability curves have been represented as thin black lines.
TABLE III. Values of $R_{m c}, \Gamma_{c}$ and $\varepsilon_{c}$ for different azimuthal velocity profiles $\hat{v}(r)=\varepsilon_{c} v_{n}(r)$ and a poloidal streamfunction $\Psi=r(1-r)^{2}$.

\begin{tabular}{cccc}
\hline \hline$v_{n}(r)$ & $R_{m c}$ & $\Gamma_{c}$ & $\varepsilon_{c}$ \\
\hline$r\left(1-r^{2}\right)$ & 131 & 0.763 & 0.36 \\
$r(1-r)$ & 112 & 0.791 & 0.36 \\
$r\left(1-r^{1 / 2}\right)$ & 105 & 0.812 & 0.362 \\
$r\left(1-r^{1 / 3}\right)$ & 103 & 0.820 & 0.365 \\
$r\left(1-r^{-1 / 2}\right)$ & 99 & 0.876 & 0.405 \\
$r(1-r)^{2}$ & 85 & 0.907 & 0.463 \\
\hline
\end{tabular}

than the result obtained in the previous subsection with the poloidal circulation (54).

In order to optimize the poloidal velocity we shall point out the main differences between the radial profiles given in (50) and (54) and drawn in Figs. 2(a) and 2(b). One of them concerns the slope $d \hat{w} / d r$ on $r=0$, which is steeper for the profile defined in (54) so as $\hat{w}$ sharply decreases to vanish at $r_{0}=0.5$. Moreover, the return flow that occurs for $r>r_{0}$ is very weak. Another difference concerns the maximum of $\hat{u}$ that arises close to the cylinder axis $(r=1 / 3)$ and is half its value in (50). These qualitative features are appreciated more quantitatively by evaluating $\bar{U}_{\text {pol2 }}=0.06$ for (54), which appears to be much lower than $\bar{U}_{\text {pol1 }}=0.148$ calculated for $(50)$. The poloidal circulation (50) is characterized by a weak mean flow concentrated in the central region of the cylinder $(r<0.5)$, a peculiarity that seems to delay dynamo action.

\section{Poloidal circulation with $\Psi=r\left(1-r^{2}\right)$}

The poloidal circulation to be considered now satisfies the requirement that $d \hat{w} / d r=0$ on $r=0$ to ensure a smooth behavior on the cylinder axis, but the condition $\hat{w}(1)=0$ has been relaxed so that

$$
\begin{aligned}
& \hat{u}(r)=-\frac{\pi}{2} r\left(1-r^{2}\right), \\
& \hat{w}(r)=\left(1-2 r^{2}\right)
\end{aligned}
$$

[see Fig. 2(c)]. In association with different toroidal velocity profiles, it leads to the values of $R_{m c}$ and $\Gamma_{c}$ shown in Table IV. The corresponding plot of $R_{m c}\left(\Gamma_{c}\right)$ in Fig. 8 is labeled with square symbols. The threshold for dynamo action is minimum when $\hat{v}(r)=\varepsilon v_{6}(r)$ and corresponds to $R_{m c}=66$, $\Gamma_{c}=0.71$. This improves over the result found with the po-

TABLE IV. Values of $R_{m c}, \Gamma_{c}$ and $\varepsilon_{c}$ for different azimuthal velocity profiles $\hat{v}(r)=\varepsilon_{c} v_{n}(r)$ and a poloidal streamfunction $\Psi=r\left(1-r^{2}\right)$.

\begin{tabular}{crll}
\hline \hline$v_{n}(r)$ & $R_{m c}$ & \multicolumn{1}{c}{$\Gamma_{c}$} & \multicolumn{1}{c}{$\varepsilon_{c}$} \\
\hline$r$ & 137 & 0.598 & 1.91 \\
$r\left(1-r^{9}\right)$ & 70 & 0.685 & 1.550 \\
$r\left(1-r^{5}\right)$ & 66 & 0.71 & 1.497 \\
$r\left(1-r^{3}\right)$ & 67 & 0.733 & 1.472 \\
$r\left(1-r^{2}\right)$ & 70 & 0.747 & 1.472 \\
$r(1-r)$ & 82 & 0.754 & 1.515 \\
\hline \hline
\end{tabular}


TABLE V. Values of $R_{m c}, \Gamma_{c}$ and $\varepsilon_{c}$ for different azimuthal velocity profiles $\hat{v}(r)=\varepsilon_{c} v_{n}(r)$ and a poloidal streamfunction $\Psi=r(1-r)^{2}(1+2 r)$.

\begin{tabular}{cccc}
\hline \hline$\hat{v}(r)$ & $R_{m c}$ & $\Gamma_{c}$ & $\varepsilon_{c}$ \\
\hline$r\left(1-r^{5}\right)$ & 88 & 0.702 & 0.765 \\
$r\left(1-r^{2}\right)$ & 63 & 0.748 & 0.742 \\
$r(1-r)$ & 58 & 0.776 & 0.743 \\
$r\left(1-r^{1 / 2}\right)$ & 57 & 0.795 & 0.752 \\
$r\left(1-r^{1 / 3}\right)$ & 57 & 0.803 & 0.757 \\
$r\left(1-r^{-1 / 2}\right)$ & 66 & 0.845 & 0.852 \\
\hline \hline
\end{tabular}

loidal circulation (54) though the present minimum threshold stays above the value found with (50). Calculation of $\bar{U}_{\text {pol3 }}$ $=0.243$ for the poloidal circulation (55) reveals a mean flow stronger than it is in the two former cases. The same conclusion can be drawn from examination of Figs. 2(a) and 2(b), where the profiles $\hat{u}(r)$ and $\hat{w}(r)$ associated with (55) exceed the two previous ones.

The main criticism to be addressed about poloidal circulations (50) and (55) is that they do not satisfy $\hat{w}(1)=0$, the latter case having the additional property that $\hat{w}(1)=-\hat{w}(0)$. The corollary is the existence of a strong return flow near the cylindrical wall that is not entirely expected in the vicinity of a solid boundary at rest. The following choice will remedy this situation.

\section{E. Poloidal circulation with $\Psi=r(1-r)^{2}(1+2 r)$}

A more realistic velocity field is obtained by imposing simultaneously no-slip boundary conditions on the cylinder $\hat{u}(1)=\hat{w}(1)=0$, and a smooth behavior on $r=0$ with $d \hat{w} / d r$ $=0$. This is the case with

$$
\begin{aligned}
& \hat{u}(r)=-\frac{\pi}{2 L} r(1-r)^{2}(1+2 r), \\
& \hat{w}(r)=(1-r)\left(1+r-5 r^{2}\right), \\
& \hat{v}(r)=4 \varepsilon r(1-r)
\end{aligned}
$$

[see Fig. 2(d)]. It is found that (56) leads to $R_{m c}=58$ for $\varepsilon_{c}$ $=0.743$ or $\Gamma_{c}=0.776$. The maximum value of the velocity occurs for $z_{\max }= \pm L / 2, r_{\max }=0$, and $U_{\max }=\hat{w}(0)=1$. Up to now, poloidal circulation (56) is the best candidate flow, giving rise to dynamo action at such a low value of $R_{m c}$. Indeed, we have slightly improved over the result obtained with the poloidal components of the flow given by (50). To understand why different poloidal circulations lead to close values of $R_{m c}$ we return for comparison to Figs. 2(a) and 2(b) where the profiles $\hat{u}(r)$ and $\hat{w}(r)$ are drawn corresponding respectively to (50) and (56). Except for the condition $\hat{w}(1)=0$, which is not satisfied in (50), the velocity profiles (50) and (56) bear more similarities with each other than with (54) or (55). In particular, in the two former cases, $\hat{u}(r)$ is maximum near the midradius, where its magnitude is nearly half the value of $\hat{w}(0)$. To check if the profile (56) can still be optimized, we performed calculations for a selection of azimuthal velocity profiles $\hat{v}(r)$ listed in Table $\mathrm{V}$ with the corresponding values of $R_{m c}$ and $\Gamma_{c}$. When plotted in Fig. 8, the
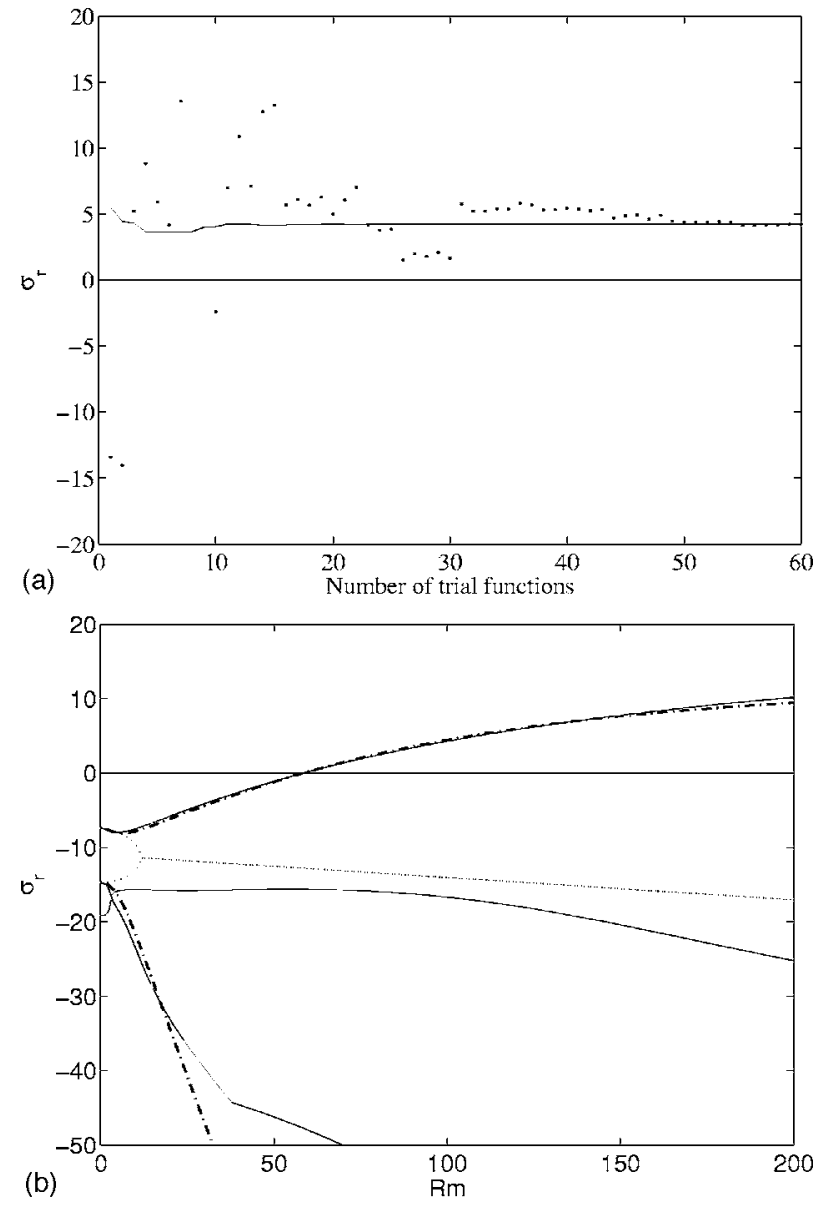

FIG. 9. (a) Real part of the leading eigenvalue obtained for the velocity field given by Eq. (56) $R_{m}=100$ and $\Gamma=0.8$, as a function of $N_{c}$, the number of trial functions used. The trial functions retained in each case are those associated to the $N_{c}$ least negative eigenvalues of the diffusion operator. Dots: Truncation of the system. Thin black line: Reduction of the system using the method developed in Sec. VI. (b) Real parts of the eigenvalues obtained for $N_{c}=2$, as a function of $R_{m}$, for the velocity field given by Eq. (56) and $\Gamma$ $=0.8$. Thin black line: Diagonalization of the full system. Dotted line: Truncation to $N_{c}=2$. Dashed-dotted line: Reduction to $N_{c}=2$.

values $R_{m c}\left(\Gamma_{c}\right)$ are labeled with diamond symbols. The threshold for dynamo action is minimum when $\hat{v}(r) \sim \varepsilon r(1$ $-r^{1 / 3}$ ) and corresponds to $R_{m c}=57$ and $\Gamma_{c}=0.803$. This is the absolute minimum found in our calculations.

\section{A METHOD OF ANALYSIS FOR LINEAR INSTABILITY MECHANISMS}

It is well known that in a variety of classical cases, such as the linear stability analyses of Rayleigh-Bénard convection or of the Couette-Taylor flow, the Galerkin approach can lead to very accurate results for even a very small number of trial functions. ${ }^{17}$ Our hope in choosing this approach was thus that, using physically motivated trial functions, we would be able to obtain good estimates of magnetic field growth rates with, if not small, at least modest numbers of trial functions. This would have allowed us to study the influences of the various flow characteristics on a small number of coupling coefficients, and thus to investigate the mechanisms of the von Kármán dynamo. We have plotted in Fig. 9 the estimate of the leading eigenvalue real part ob- 
tained in the reference case $R_{m}=100, \Gamma=0.8$, the velocity field given by Eq. (56), as a function of the number of trial functions used. We can see that, in contrast to what happens in the above-mentioned cases, this number must indeed be extremely large (at least $>50$ ) if good eigenvalue estimates are to be obtained. This can probably be ascribed to the quite complex structure of the velocity fields we have used, which leads to strong (and crucially important) coupling of the large-scale features of the magnetic field with their smallscale counterparts, which must thus be adequately represented. Since a parametric study of the coefficients of a 50 $\times 50$ matrix is clearly out of reach, we had to devise a way of reducing the size of our problem, i.e., of constructing a smaller coupling matrix possessing the same leading eigenvalues as the full one.

\section{A. Reducing the problem size}

The gist of our approximation process is the following remark: if $\mathbf{X}$ is an eigenvector of a matrix $\mathbf{M}$, and if $\sigma$ is its associated eigenvalue, we know that $(\mathbf{M}-\sigma \mathbf{I}) \mathbf{X}=\mathbf{0}$, but if $\mathbf{X}$ is an almost-neutral eigenvector of $\mathbf{M}$, and if we know $\sigma$ to be real, it may happen that $\sigma$ is negligible with respect to most of the diagonal coefficients of $\mathbf{M}$. The corresponding equations of the system effectively do not involve $\sigma$ as an unknown. This piece of information can then be exploited to reduce the order of the matrix. In our dynamo problem the matrix $\mathbf{M}$ we have to deal with corresponds to the algebraic system (45) in Sec. IV G. Its diagonal elements are $-\lambda_{k j}$. We know from their definition in (38) and from the behavior of the $\mu_{k j}$ when $j$ increases that the $\lambda_{k j}$ become increasingly large as $k$ and/or $j$ increase.

Assume $\mathbf{M}$ has been rearranged by a similarity transform in such a way that its $N_{c}$ diagonal coefficients with respect to whom $\sigma$ cannot be neglected are grouped in the upper lefthand corner. Let $\mathbf{X}_{\mathbf{c}}, \mathbf{X}_{\mathbf{s}}, \mathbf{A}, \mathbf{B}, \mathbf{C}$, and $\mathbf{D}$ partition $\mathbf{X}$ and $\mathbf{M}$ as

$$
\mathbf{X}=\left[\begin{array}{c}
\mathbf{X}_{c} \\
\mathbf{X}_{s}
\end{array}\right], \quad \mathbf{M}=\left[\begin{array}{ll}
\mathbf{A} & \mathbf{B} \\
\mathbf{C} & \mathbf{D}
\end{array}\right] .
$$

A collects the diagonal coefficients with respect to whom $\sigma$ cannot be neglected. The associated part of $\mathbf{X}, \mathbf{X}_{\mathbf{c}}$, collects the "central" trial functions amplitudes. $\mathbf{C}$ represents the forcing these central components induce on the "slaved" components $\mathbf{X}_{\mathrm{s}}$. D represents the interactions of slaved components among themselves, and $\mathbf{B}$ represents the feedback of the slaved components on the central ones. Had $\mathbf{B}$ been identically null, using in the Galerkin expansion only the trial functions associated to A would have been successful. Our aim is now to reduce the problem to a matrix the size of $\mathbf{A}$. As mentioned above, $\mathbf{X}$ and $\sigma$ are such that

$$
\begin{aligned}
& (\mathbf{A}-\sigma \mathbf{I}) \mathbf{X}_{c}+\mathbf{B} \mathbf{X}_{s}=0, \\
& \mathbf{C} \mathbf{X}_{c}+(\mathbf{D}-\sigma \mathbf{I}) \mathbf{X}_{s}=0 .
\end{aligned}
$$

Neglecting $\sigma$ in the second of these equations leads to the following expression for $\mathbf{X}_{\mathbf{s}}$ in terms of $\mathbf{X}_{\mathbf{c}}$ :

$$
\mathbf{X}_{s}=-\mathbf{D}^{-1} \mathbf{C} \mathbf{X}_{c},
$$

which is substituted in (57), leading to the reduced eigensystem involving only $\mathbf{X}_{\mathbf{c}}$ :

$$
\left(\mathbf{A}-\mathbf{B D}^{-1} \mathbf{C}\right) \mathbf{X}_{c}=\sigma \mathbf{X}_{c} .
$$

The corresponding results for the reference case are presented in Fig. 9(a). We can see that the reduced matrix possesses a leading eigenvalue that is very close to that of the full problem, even for a very small number of central trial functions. A reduction procedure using ten central eigenfunctions seems sufficient to achieve a precision better than that obtained through truncation to 50 trial functions. Figure 9(b) compares the leading eigenvalue estimates obtained through diagonalization of the full matrix with those obtained through reduction or truncation to two central trial functions. We can see that even with this small number of trial functions our approximation captures in a very satisfactory way the behavior of the leading eigenvalue of the full problem. The quality of the results actually improves as the instability threshold is approached, as the eigenvalue of interest becomes negligible with respect to more diagonal coefficients of $\mathbf{M}$. We have checked that the structure of the approximate eigenmodes constructed by complementing the central amplitudes with a slave part determined from Eq. (59) corresponds very closely to that of the full eigenmodes. ${ }^{18}$

Some comments are in order at this point:

Our approximation is very similar to the one involved in the "center-manifold reduction" often used at the nonlinear stage in bifurcation theory. ${ }^{25}$ Instead of eliminating stable eigenmodes of a linear bifurcation problem to obtain equations describing the nonlinear evolution of unstable eigenmodes beyond the instability threshold, we have chosen to eliminate "very stable" eigenmodes of our problem for $R_{m}$ $=0$ to obtain equations describing their effect on the linear evolution of "less stable" eigenmodes for finite values of $R_{m}$. The essential mechanism used is however the same: the slaved trial functions are so strongly damped that their amplitudes are set by the balance between forcing from the central trial functions and the damping. Perturbations away from this balance decay very fast, with a time scale associated with ohmic diffusion. Each central trial function, away from $R_{m}=0$, thus carries along its path a host of slave trial functions, whose amplitudes adjust instantaneously to any change in the central amplitude, provided it is sufficiently slow. It is thus clear how our full problem can be reduced to an equivalent, smaller one, involving only central trial function amplitudes.

Although we have concentrated our exposition on eigenvalues close to zero, it is clear that our method can be transposed in a straightforward way to other situations, by merely adding to the full matrix the identity matrix, multiplied by a rough estimate of the eigenvalue of interest.

As in any approximation procedure, a considerable degree of arbitrariness is involved in the choice of the central trial functions. Too low a value of $N_{c}$ may lead to large imprecision in the approximated value of $\sigma$, while too large a 
value may lead to an unnecessarily large reduced eigenproblem, which may hamper the study of the instability mechanism.

Although Fig. 9 shows that our approximation process allows us to construct a reduced matrix that possesses eigenvalues much closer to those of the full problem than those obtained through truncation, we must stress that the construction of the reduced matrix is still computationally quite intensive for the two following reasons: first, it is clear that the elementary matrices used in the computation of the reduced problem change with $R_{m}$. The computation must thus be repeated for each set of control parameter values. Second, though the reduced problem may be very small, a large initial matrix must still be constructed in order to take properly into account the small-scale structure of the magnetic field. Our method minimizes the amount of work needed in the actual diagonalization process, but the construction of the large problem must still be performed completely. The point is, however, that the diagonalization process itself is the most computationally intensive task, and is also the part in which the instability mechanism we want to analyze is involved.

\section{B. Study of the reduced problem coefficients for the reference case}

We will now study the matrix obtained by reduction of the full problem to the interaction of the two trial functions associated to the least negative eigenvalues of the diffusion operator. The first of these functions, $\boldsymbol{\Psi}_{\mathbf{c}}^{\mathbf{1}}$, corresponds to a magnetic dipole, centered on the origin of coordinates of Fig. 6 , antisymmetric with respect to $\mathbf{R}_{\mathbf{L}}$, pointing in the $Y$ direction. It is responsible for the dipolar component of the magnetic field eigenmode apparent in Fig. 6(e). The second central trial function, $\boldsymbol{\Psi}_{\mathbf{c}}^{\mathbf{2}}$, is the first trial function that possesses a $k=0$ axial dependency. Its only non-null component points in the $Z$ direction. It is responsible for the magnetic field "annulus" that seems to surround the origin of coordinates in Fig. 6(c). We will use throughout this study the transparent notation $r_{11}, r_{12}, r_{21}, r_{22}$ for the elements of the reduced interaction matrix. Straightforward algebra shows that the leading eigenvalue of the reduced matrix reads

$$
\sigma=\frac{\sqrt{\left(r_{11}-r_{22}\right)^{2}+4 r_{12} r_{21}}+r_{11}+r_{22}}{2} .
$$

Clearly, instability will set in most easily in cases where the off-diagonal terms $r_{12}$ and $r_{21}$ are of the same sign, and where the sum of the diagonal terms is not too negative. With this in mind, we will as a first step study the behavior of the coupling coefficients in the neighborhood of the instability threshold in the reference case of velocity field (56). The various coefficients are plotted as a function of $R_{m}$ in Fig. 10 (a) for $\Gamma=0.8$. We can see that, for large enough $R_{m}, r_{12}$ and $r_{21}$ are indeed both negative, which is fortunate since both diagonal terms appear to be quite strongly negative. The behavior of the various coefficients seems at first sight monotonously decreasing in most of the range of interest. Deviations from linearity exist, but their amplitudes seem to depend quite smoothly on $R_{m}$. This smooth behavior is to be contrasted with the quite rapid evolutions revealed by closer
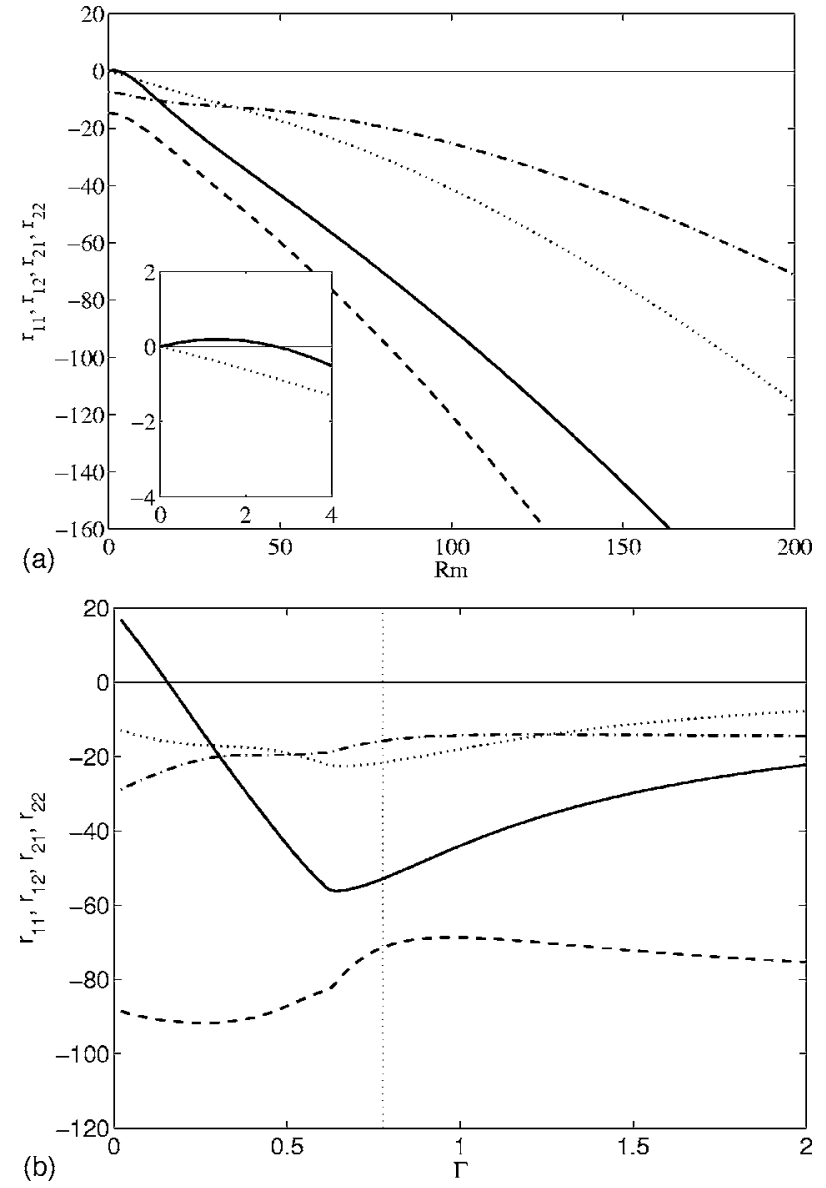

FIG. 10. Coefficients of the reduced coupling matrix, obtained for the velocity field given by Eq. (56) as a function of (a) $R_{m}$, for $\Gamma=0.8$. (b) $\Gamma$, for $R_{m}=60$. Dashed-dotted line: $r_{11}$. Dotted line: $r_{12}$. Continuous line: $r_{21}$. Dashed line: $r_{22}$. In the bottom graph, the optimal value of $\Gamma$ is marked as a thin vertical dotted line.

inspection of the curves in the vicinity of $R_{m}=0$. Indeed, one can see that close to $R_{m}=0$, the coefficient $r_{21}$ starts by increasing slightly, reaches a maximum value, and decreases in the remainder of the range. As before, this shows that careless truncation of the system to too few trial functions can only lead to erroneous results as regards the leading eigenvalues of the linear stability problem. The coefficients of the reduced matrix have been plotted as a function of $\Gamma$ for $R_{m}$ $=60$ in Fig. 10(b). This value of $R_{m}$ is close to the threshold value, which implies that $\sigma$ can indeed be considered negligible where necessary. Although $r_{11}, r_{12}$, and $r_{22}$ display as before rather monotonic behaviors, we can see that $r_{21}$ has a very sharp minimum for a value of $\Gamma$ quite close to that for which the threshold value of the magnetic Reynolds number is optimal. The other coefficients also seem affected in that range of values of $\Gamma$, but the induced perturbations remain of considerably lesser amplitude. Going back to the definition of $r_{21}$, we can see that this coefficient describes the coupling of the central trial function $\boldsymbol{\Psi}_{\mathbf{c}}^{\mathbf{1}}$, and an account taken of its slaved functions, with $\boldsymbol{\Psi}_{\mathbf{c}}^{\mathbf{2}}$. It thus characterizes the efficiency of the mechanism responsible for conversion of the dipolar component of the magnetic field into an axially directed magnetic field located in the perpendicular plane. Pétrélis et $a l^{26}$ considered such a conversion mechanism to be akin to 

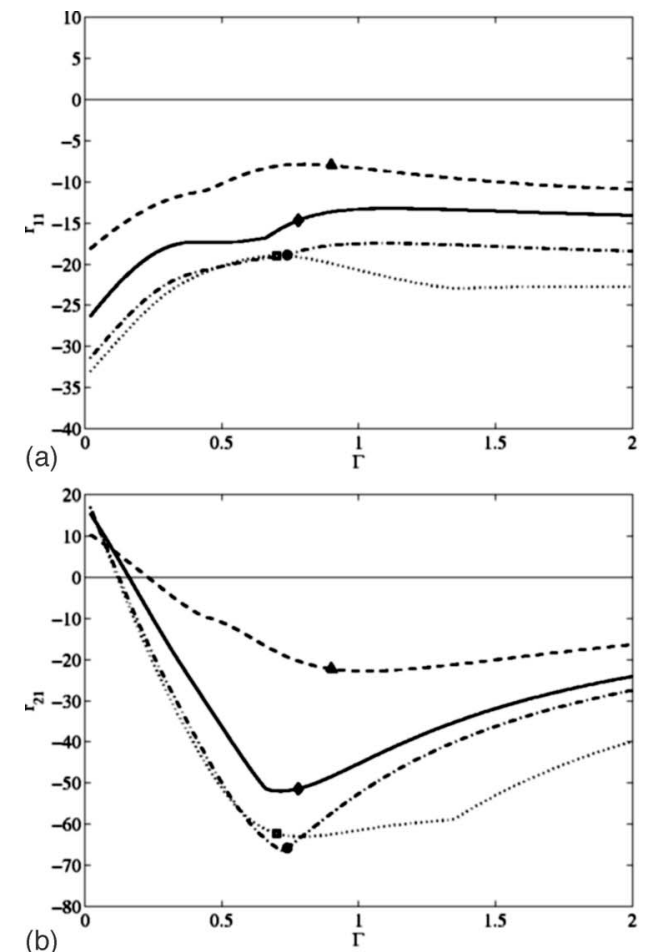

(b)
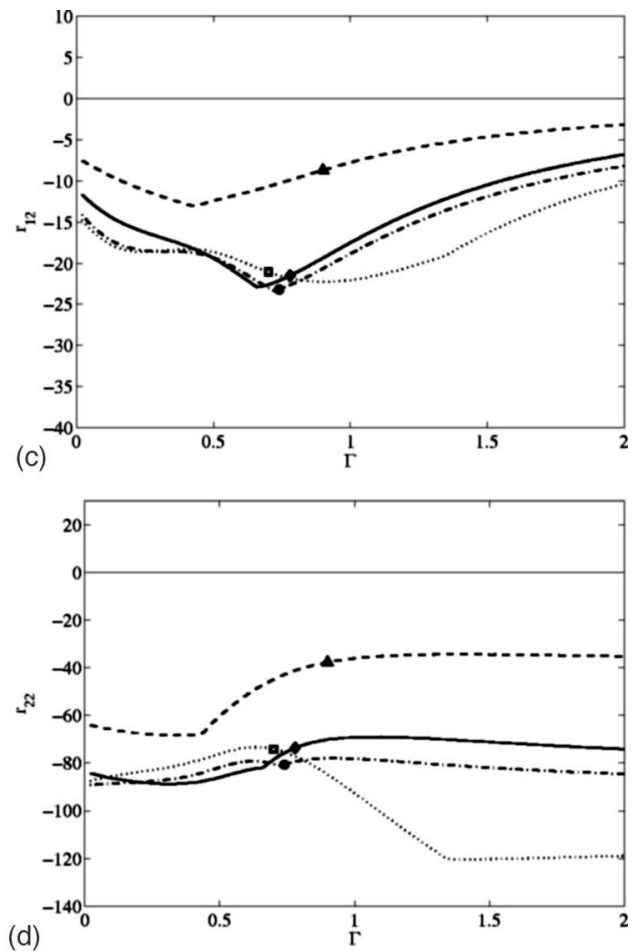

FIG. 11. Coefficients of the reduced coupling matrices as functions of $\Gamma$ for $R_{m}=R_{m c}$. Dashed-dotted line: $\Psi=r(1$ $-r), v(r)=r\left(1-r^{2}\right), R_{m}=60$. Dashed line: $\Psi=r(1-r)^{2}, v(r)=r(1-r)^{2}, R_{m}$ $=85$. Dotted line: $\Psi=r\left(1-r^{2}\right), v(r)$ $=r\left(1-r^{5}\right), R_{m}=66$. Continuous line: $\Psi=r(1-r)^{2}(1+2 r), \quad v(r)=r\left(1-r^{1 / 3}\right)$, $R_{m}=60$. In each case, the optimal value of $\Gamma$ has been marked with the symbol appropriate to $\Psi$. (a) $r_{11}$, (b) $r_{21}$, (c) $r_{12}$, (d) $r_{22}$. the " $\alpha$ effect" originally introduced by Parker. ${ }^{27}$ Indeed, we have checked that the sign of $r_{21}$ changes as the direction of rotation of the impellers changes. This shows that $r_{21}$ behaves as a pseudoscalar under mirror symmetries, such as the velocity field helicity that is often invoked in this context. Following Leprovost, ${ }^{28}$ the mean helicity when normalized to the mean velocity is a function of the poloidal-to-toroidal ratio $\Gamma$ that reaches its maximum value for a particular $\Gamma_{h}$ corresponding to equipartition of the toroidal and poloidal kinematic energy. When calculated for our model flows, there is no close correlation between the value $\Gamma_{h}$ and the value $\Gamma_{c}^{*}$ for which the minimum of $R_{m c}(\Gamma)$ occurs.

The transformation of axial to dipolar magnetic field, of which $r_{12}$ is a measure, can easily be interpreted as distortion of the axial field by the toroidal velocity field, i.e., an " $\Omega$ effect." This effect remains of quite constant amplitude throughout the range in $\Gamma$, which could be considered quite surprising since it is intuitively associated to the effect of the toroidal flow alone. Only in the limit of large values of $\Gamma$ does $r_{12}$ decrease significantly. The fact that this effect is not significantly stronger for large toroidal velocity fields may indicate that the tail of slaved functions of $\boldsymbol{\Psi}_{\mathrm{c}}^{\mathbf{2}}$ changes structure. However, the fact that the growth rate of the magnetic field depends crucially on the product of $r_{12}$ and $r_{21}$ leads us to propose the von Kármán kinematic dynamo as an " $\alpha \Omega$ dynamo." 29

\section{Comparison with the reduced matrix coefficients for the other flows}

For each of the four poloidal circulations presented in Sec. V, we have plotted against $\Gamma$ in Fig. 11 the coefficients of the reduced matrix associated to the best toroidal circulation tested. In each case, the value of $R_{m}$ has been chosen close to the threshold value. A striking point is that in all cases $r_{21}$ presents a rather sharp minimum for a value of $\Gamma$ quite close to the optimum. We can see that the conversion from bipolar field ( $\psi_{c}^{1}$ component) to axial field ( $\psi_{c}^{2}$ component) is much more efficient for the poloidal circulations associated with Eqs. (50) and (55) than for poloidal circulation (54). The case of poloidal circulation (56), although it happens to have the lowest threshold value of $R_{m}$, lies in between. The same remark also applies to $r_{12}$, though here poloidal circulation (56) achieves results similar to those of (50) and (55). The cusps that can be observed on the various curves stem from our definition of the magnetic Reynolds number. We have checked, however, that the minimum of $r_{21}$ is still observed when we use a definition based on an integral measure of the flow magnitude, and coincides actually better with the optimal value of $\Gamma$. As $r_{21}$ achieves its largest values for intermediate $\Gamma$, we expect the effect it describes to involve both the poloidal and the toroidal circulations. We have left the complete study of such complex processes for future work.

The remaining coefficients present less striking features. The coefficient $r_{11}$ represents the effect of the flow on the self-interaction of $\psi_{c}^{1}$ with itself. It is always quite strongly negative, which means that the flow tends to enhance the diffusion of the dipolar component of the magnetic field. The sharp increase in absolute value of $r_{11}$ when $\Gamma$ tends to 0 (limit of vanishing poloidal circulation) shows that the toroidal velocity plays an important role in this effect. Expulsion of an externally imposed dipolar magnetic field by a toroidal vorte ${ }^{30}$ has been documented in a liquid gallium experiment by Odier $e t a l .{ }^{31}$ The coefficient $r_{11}$ seems to possess a welldefined limit when the poloidal velocity becomes dominant. This limit is dependent on the poloidal circulation consid- 
ered, and probably also depends on the magnetic Reynolds number. It could be understood as expressing the balance between the dipolar magnetic field stretching effect of the poloidal flow convergence region located near the cylinder wall at $r=1, z=0$, and the "compression" effect of the central stagnation point. The distributions of inward radial velocities represented in Fig. 2 fit nicely in this picture. We can see that, the closer the maximum of $\hat{u}(r)$ lies to the axis of the cylinder, the less extended the stagnation point region is, and the less negative is the value of $r_{11}$ for large $\Gamma$. This may explain the low threshold value obtained with poloidal circulation (56), which performs quite modestly as regards the magnetic field amplification, but whose associated values of $r_{11}$ are not too strongly negative. A toy poloidal circulation obtained by multiplying the streamfunction of velocity field (56) by $r$, and whose stagnation point influence is thus much smaller, is associated with values of $r_{11}$ in the large $\Gamma$ limit actually less negative than those linked with poloidal circulation (54), showing our interpretation of this effect to be fairly sound.

The other diagonal coefficient, $r_{22}$, which corresponds to the flow-induced dissipation of axial magnetic field, achieves values more negative by a factor of nearly 4 . Except in the case of poloidal circulation (54), a larger poloidal circulation does not seem to slow down this effect. Indeed, poloidal circulation (55) seems to accelerate the dissipation. This is quite unexpected, as the stagnation point located at the center of the flow should be more efficient at stretching the magnetic field lines in this limit. An explanation for this behavior could be that the quite intense inward radial velocities that converge to the center of the flow bring close together the two regions of opposite axial field located on either side of the axis of the cylinder, and hence accelerate diffusion. This is quite consistent with the fact that poloidal circulation (54), which possesses weak radial velocities associated to an intense axial flow of small extent, seems to dissipate less in the large $\Gamma$ limit. The toy poloidal circulation mentioned in the previous paragraph also possesses less negative values of $r_{22}$, quite close to those achieved by poloidal circulation (54).

The last coefficient, $r_{12}$, which we associate with the $\Omega$ effect in our flow, behaves in a more intuitive way. Starting from a limiting value for small $\Gamma$ (i.e., small poloidal circulation), its magnitude gradually increases before decreasing to zero for the vanishing toroidal velocity field intensity. It reaches comparable magnitudes for all the velocity fields studied, with the notable exception of poloidal circulation (54).

Poloidal circulation (54) is somewhat special, achieving self-excitation mainly by being inefficient in many respects. Close scrutiny of the various curves shows that it achieves very poor performances as regards magnetic field amplification, but that this is compensated by its lack of strong flowinduced dissipation. The various arguments presented above ascribe this to the very limited extent of the region of strong flow amplitude. We wish to stress that there is something more profound to this than the mere need to define the magnetic Reynolds number using an integral estimate of the velocity scale rather than the maximum value achieved in the flow volume. The point here is that changing the definition of the magnetic Reynolds number would alter its value at threshold, but not the amplitudes of the various interaction coefficients. The self-excitation mechanism of poloidal circulation (54) involves a balance between weak amplification and weak dissipation, whereas the self-excitation mechanisms of the other flows involve balances between strong amplification and a large dissipation. This holds regardless of the magnetic Reynolds number definition. The effect on the kinematic dynamo effect of large areas of slow motion surrounding the active part of the flow has been studied specifically in Ref. 13.

\section{CONCLUSION AND REMARKS}

The Galerkin method implemented here to solve the induction equation in a cylindrical geometry is a good complementary approach to numerical time integration. Though time integration seems to be the method of choice when the temporal behavior of the magnetic field is expected to be complex and not easily predictable, we consider direct diagonalization to be more appropriate in the case of the linear stability analysis of a time-independent configuration.

The Galerkin method is particularly suitable for determining the dynamo threshold since it provides the critical value of the magnetic Reynolds number faster than numerical time integration. This allows one to test a large number of model velocity profiles in order to select the best candidate for dynamo action. Contrary to the approach followed in Ref. 12 , where experimental mean velocity profiles were used to compute the critical magnetic Reynolds number, we prospected model flows chosen to exhibit spatial characteristics whose role in dynamo action we wanted to assess. Starting with a rough model flow, we have gradually modified its main characteristics in order to obtain a more elaborate flow, able to produce dynamo at a low threshold value $\left(R_{m c}=57\right)$. This model flow differs in a significant way from those observed in experiments, but we hope it nevertheless provides a good direction in the design of future experimental setups.

Another advantage of the Galerkin approach is that it allows one to take advantage of the flow symmetries and their subsequent influence on the spatial and temporal properties of the magnetic field resulting from dynamo action. Arguing about the symmetry with respect to the midheight plane $(z=0)$, it is first deduced that the bifurcation will be towards either stationary modes $\left(\sigma_{i}=0\right)$ or oscillatory modes with a complex conjugate growth rate. We have then shown that the symmetry of the flow with respect to its end boundaries $(z= \pm L)$ is responsible for the separation in two types of modes that have distinct spatial properties, which we referred to as symmetric or antisymmetric modes. As a result of our calculations it appears that the preferred mode of magnetic field at the dynamo threshold is always a stationary antisymmetric mode for all the tested velocity profiles.

In the frame of dynamo theory, attention has focused on Beltrami flows, in which the vorticity is a constant multiple of the velocity, as these flows are believed to give rise to fast dynamos. A typical example is the steady ABC flow, which is periodic in the three directions of space. ${ }^{9}$ In cylindrical geometry, Beltrami flows are also encountered among the flows 
that are periodic along the cylinder axis. Assuming their poloidal circulation has the same symmetry properties and periodicity as in von Kármán flows, such Beltrami flows are associated with toroidal velocities that behave like $\sin (\pi z / L)$ in the axial direction. The reflection symmetry of the von Kármán flow about the end boundaries $z= \pm L$ is replaced by a rotational symmetry of the type of $R_{\pi}$ about these boundaries. We can thus expect some of our general results to hold for these flows of widespread interest, namely that the magnetic field modes will still be stationary or associated to complex-conjugate growth rates, and that the decomposition in modes symmetric and antisymmetric with respect to rotations about the end boundaries will still apply.

More generally, our Galerkin analysis can easily take advantage of the symmetry properties of flow configurations, and appears to be a powerful tool to solve the kinematic dynamo problem.

We will now present critical remarks to show possible directions that may deserve further study.

In the case where only one central function is considered, our reduction process corresponds to a single iteration of the inverse power method, carried out on the full matrix $\mathbf{M}$, using the central trial function as a first guess. Reduction to a central space spanned by a larger number of trial functions followed by diagonalization of the remaining matrix can likewise be considered as one step of a Krylov-spacebased diagonalization method carried out on the inverse of M (see, e.g., Ref. 32 for more details on Krylov methods). Our derivation, by providing a clear physical picture of the mechanism underlying such diagonalization schemes, should provide a rationale for the introduction of physically motivated preconditioners, which could greatly improve convergence rates and accuracies in a wide variety of applications.

Although we have chosen to construct the full problem using a Galerkin method, other approaches could be used and lead to a nearly identical reduced matrix. Equation (59), for instance, merely expresses the fact that the full eigenmode must lie in the span of the inverses of the central trial functions. These inverses can be computed using whatever discretization scheme seems appropriate to the problem at hand, and actually in some cases analytically. The reduced eigensystem (60) expresses the fact that the effect of the operator on the eigenvector must be to multiply its projections on the central weighting functions by $\sigma$. Though construction of the central weighting functions does require some preliminary work, it can without doubt be performed for any given discretization scheme. We are thus confident that our method is of quite general applicability, and that it could provide a valuable analysis tool for the numerous areas of physics where the mechanisms governing the dynamics of eigenmodes need to be understood and quantified.

We admit our attempt to relate the properties of the various coupling coefficients to the features of the flow at hand is still very preliminary. Analytical computation of the reduced matrix coefficients is probably feasible in our case (see, e.g., Ref. 33 for a study of the Green's function of the induction operator), and would be a very welcome help in this task. Such a computation could in particular help in settling the longstanding issue of the link between $\alpha$-effect efficiency and the kinetic helicity of the velocity field, and at long last take optimization of dynamo-related experimental setups past the trial-and-error stage.

\section{ACKNOWLEDGMENTS}

We wish to thank J. Léorat, who made possible the comparison with numerical simulations, and L. Tuckerman for fruitful discussions about symmetry. We thank A. Fondin, who contributed to this work during her training. We also greatly appreciated stimulating discussions with A. Chiffaudel, B. Dubrulle, N. Leprovost, and F. Ravelet.

\section{APPENDIX: EXPRESSION OF THE QUANTITIES INVOLVED IN THE RESIDUALS}

The quantities $\hat{T}_{m}^{k j}$ introduced in Sec. IV E are expressed as follows:

$$
\begin{aligned}
& \hat{T}_{m \pm 1}^{k j}=\operatorname{sgn}(k) S_{ \pm}^{k j} \mp \frac{\pi \hat{v}}{2 L} \psi_{m}^{k j}(r), \\
& \hat{T}_{m}^{k j}=m \frac{\hat{v}}{r} \psi_{m}^{k j}(r),
\end{aligned}
$$

while the expressions for $\hat{P}_{m}^{k j}$ are given below:

$$
\begin{aligned}
& \hat{P}_{m \pm 1}^{k j}=\operatorname{sgn}(k) \mathcal{P}_{m \pm 1}^{k j}-\frac{\pi \hat{u}}{L} \psi_{m}^{k j}(r), \\
& \hat{P}_{m}^{k j}=\left(\hat{u} \frac{\partial}{\partial r}+(k-2) \frac{\pi \hat{w}}{2 L}\right) \psi_{m}^{k j}(r)+\operatorname{sgn}(k) \mathcal{P}_{m}^{k j},
\end{aligned}
$$

where

$$
\begin{aligned}
& \mathcal{P}_{m \pm 1}^{k j}=C_{ \pm}^{k j}+\frac{k \pi}{2 L} \hat{w} \psi_{m \pm 1}^{k j}(r), \\
& \mathcal{P}_{m}^{k j}=-\frac{1}{2} \frac{d \hat{w}}{d r}\left(\psi_{m+1}^{k j}(r)+\psi_{m-1}^{k j}(r)\right),
\end{aligned}
$$

with $C_{ \pm}^{k j}=C_{ \pm}\left(\psi_{m \pm 1}^{k j}, \hat{u}\right), S_{ \pm}^{k j}=S_{ \pm}\left(\psi_{m \pm 1}^{k j}, \hat{v}\right)$.

When $k=0$, the expressions for $\mathcal{P}_{m \pm 1}^{0 j}$ and $\mathcal{P}_{m}^{0 j}$ are readily deduced from the above general expressions (A5) and (A6) while the other quantities take the following specific values:

$$
\begin{aligned}
& \hat{P}_{m \pm 1}^{0 j}=-\frac{\pi}{L} \hat{u} \psi_{m}^{0 j}, \quad \hat{P}_{m}^{0 j}=\left(\hat{u} \frac{\partial}{\partial r}-\frac{\pi}{L} \hat{w}\right) \psi_{m}^{0 j}, \\
& \hat{T}_{m \pm 1}^{0 j}=\mp \frac{\pi}{2 L} \hat{v} \psi_{m}^{0 j}, \quad \hat{T}_{m}^{0 j}=m \frac{\hat{v}}{r} \psi_{m}^{0 j}, \\
& \mathcal{T}_{m \pm 1}^{0 j}=S_{ \pm}^{0 j}, \quad \mathcal{T}_{m}^{0 j}=0 .
\end{aligned}
$$

${ }^{1}$ Sir J. Larmor, "How could a rotating body such as the sun become a magnet?" Report of the 87th Meeting of the British Association for the Advancement of Science, Bournemouth (John Murray, London, 1919), pp. $159-160$.

${ }^{2}$ Y. B. Ponomarenko, "Theory of the hydromagnetic generator," J. Appl. Mech. Tech. Phys. 6, 755 (1973).

${ }^{3}$ A. Gailitis, O. Lielausis, S. Dement'ev, E. Placatis, A. Cifersons, G. Gerbeth, T. Gundrum, F. Stefani, M. Christen, H. Hanel, and G. Will, "Detection of a flow induced magnetic field eigenmode in the Riga dynamo 
facility," Phys. Rev. Lett. 84, 4365 (2000).

${ }^{4}$ R. Stieglitz and U. Muller, "Experimental demonstration of a homogeneous two-scale dynamo," Phys. Fluids 13, 561 (2001).

${ }^{5}$ G. O. Roberts, "Dynamo action of fluid motions with two-dimensional periodicity," Philos. Trans. R. Soc. London, Ser. A 271, 411 (1972).

${ }^{6}$ N. L. Peffley, A. B. Cawthrone, and D. P. Lathrop, "Toward a selfgenerating magnetic dynamo: The role of turbulence," Phys. Rev. E 61, 5287 (2000).

${ }^{7}$ L. Marié, J. Burguete, A. Chiffaudel, F. Daviaud, D. Ericher, C. Gasquet, F. Pétrélis, S. Fauve, M. Bourgoin, M. Moulin, P. Odier, J.-F. Pinton, A. Guigon, J. B. Luciani, F. Namer, and J. Léorat, "MHD in von Kármán swirling flows, development and first run of the VKS experiment," in Dynamo and Dynamics, A Mathematical Challenge, Proceedings of the NATO Advanced Research Workshop, Cargèse (France), 21-26 August 2000, NATO Science Series II, edited by P. Chossat, D. Armbruster, and I. Oprea (Kluwer Academic, Dordrecht, The Netherlands, 2001), Vol. 26, pp. $35-50$.

${ }^{8}$ M. Bourgoin, L. Marié, F. Pétrélis, C. Gasquet, A. Guignon, J.-B. Luciani, M. Moulin, F. Namer, J. Burgete, A. Chiffaudel, F. Daviaud, S. Fauve, P. Odier, and J.-F. Pinton, "Magnetohydrodynamics measurements in the von Kármán sodium experiment," Phys. Fluids 14, 3046 (2002).

${ }^{9}$ D. J. Galloway and M. R. E. Proctor, "Numerical calculations of fast dynamos in smooth velocity fields with realistic diffusion," Nature (London) 356, 691 (1992).

${ }^{10}$ C. Normand, "Ponomarenko dynamo with time-periodic flow," Phys. Fluids 15, 1606 (2003).

${ }^{11}$ C. Nore, M.-E. Brachet, H. Politano, and A. Pouquet, "Dynamo action in the Taylor-Green vortex near threshold," Phys. Plasmas 4, 1 (1997).

${ }^{12}$ L. Marié, J. Burguete, F. Daviaud, and J. Léorat, "Numerical study of homogeneous dynamo based on experimental von Kàrmàn type flows," Eur. Phys. J. B 33, 469 (2003).

${ }^{13}$ F. Ravelet, A. Chiffaudel, F. Daviaud, and J. Léorat, "Towards a von Kármán dynamo: Numerical studies based on experimental flows," Phys. Fluids 17, 117104 (2005).

${ }^{14}$ N. L. Dudley and R. W. James, "Time-dependent kinematic dynamos with stationary flows," Proc. R. Soc. London, Ser. A 425, 407 (1989).

${ }^{15}$ P. W. Livermore and A. Jackson, "On magnetic energy instability in spherical stationary flows," Proc. R. Soc. London, Ser. A 460, 1453 (2004).

${ }^{16}$ T. G. Cowling, "The magnetic field of sunspots," Mon. Not. R. Astron. Soc. 94, 39 (1934).

${ }^{17}$ S. Chandrasekhar, Hydrodynamic and Hydromagnetic Stability (Claren- don, Oxford, 1961).

${ }^{18} \mathrm{~L}$. Marié, "Transport de moment cinétique et de champ magnétique par un écoulement tourbillonnaire turbulent: influence de la rotation," Thèse de doctorat de l'Université Paris 7 (2003).

${ }^{19}$ C. Nore, L. S. Tuckerman, O. Daube, and S. Xin, "The 1:2 mode interaction in exactly counter-rotating von Kàrmàn swirling flow,” J. Fluid Mech. 477, 51 (2003).

${ }^{20} \mathrm{E}$. Knobloch, "Symmetry and instability in rotating hydrodynamic and magnetohydrodynamic flows," Phys. Fluids 8, 1446 (1996).

${ }^{21}$ G. E. Backus, "A class of self-sustaining dissipative spherical dynamos," Ann. Phys. (N.Y.) 4, 372 (1958).

${ }^{22}$ P. M. Morse and H. Feschbach, Methods of Theoretical Physics, Part 1 (McGraw-Hill, New York, 1953).

${ }^{23}$ R. Kaiser and A. Tilgner, "Kinematic dynamos surrounded by a stationary conductor," Phys. Rev. E 60, 2949 (1999).

${ }^{24}$ B. A. Finlayson, The Method of Weighted Residuals and Variational Principles (Academic, New York, 1972).

${ }^{25}$ A. H. Nayfeh and B. Balachandran, Applied Nonlinear Dynamics, Analytical, Computational and Experimental Methods (Wiley-Interscience, New York, 1994).

${ }^{26}$ F. Pétrélis, M. Bourgoin, L. Marié, J. Burguete, A. Chiffaudel, F. Daviaud, S. Fauve, P. Odier, and J.-F. Pinton, "Nonlinear magnetic induction by helical motion in a liquid sodium turbulent flow," Phys. Rev. Lett. 90, 174501 (2003).

${ }^{27}$ E. N. Parker, "Hydromagnetic dynamo models," Astrophys. J. 163, 255 (1955).

${ }^{28} \mathrm{~N}$. Leprovost, "Influence des petites échelles sur la dynamique à grande échelle en turbulence hydro et magnétohydrodynamique," Thèse de doctorat de l'Université Paris 6 (2004).

${ }^{29}$ L. Marié, F. Pétrélis, M. Bourgoin, J. Burguete, A. Chiffaudel, F. Daviaud, S. Fauve, P. Odier, and J.-F. Pinton, "Open questions about homogeneous fluid dynamos: the VKS experiment," Magnetohydrodynamics 38, 156 (2002).

${ }^{30}$ H. K. Moffatt, Magnetic Field Generation in Electrically Conducting Fluids (Cambridge University Press, Cambridge, 1978).

${ }^{31}$ P. Odier, J.-F. Pinton, and S. Fauve, "Magnetic induction by coherent vortex motion," Eur. Phys. J. B 16, 373 (2000).

${ }^{32}$ Y. Saad, Numerical Methods for Large Eigenvalue Problems (Halstead, Broadway, Australia, 1992).

${ }^{33}$ W. Dobler and K.-H. Rädler, "An integral equation approach to kinematic dynamo models," Geophys. Astrophys. Fluid Dyn. 89, 45 (1998). 